RADIATION PHYSICS, BIOPHYSICS, AND RADIATION BIOLOGY

Progress Report for the Period December 1, 1991-November 30, 1992

By

E. J. Hall

M. Zaider

May 1992

Work Pertormed Under Contract No. FG02-88E?:60631

College of Physicians \& Surgeons of Columbla University New York, New York 


\title{
RADIATION PHYSICS, BIOPHYSICS, AND RADIATION BIOLOGY
}

\author{
Progress Report
}

For Period December 1, 1991-November 30, 1992

E.J. Hall, Principal Investigator

M. Zaider, Co-Principal Investigator

Center for Radiological Research

Department of Radiation Oncology

College of Physicians and Surgeons of Columbia University

May, 1992

Prepared for

THE U.S. DEPARTMENT OF ENERGY

UNDER GRANT NO. DE-FG02-88ER60631 


\section{TABLE OF CONTENTS}

Page

INTRODUCTION

STAFF NEWS

THE COLUMBIA COLLOQUIUM

RESEARCH STAFF

6

TEACHING

RADIATION PHYSICS AND MICRODOSIMETRY

Design and Development of a New Wall-Less Ultra Miniature

Proportional Counter for Nanodosimetry

P. Kliauga

Some Recent Measurements of Ionization Distributions for Heavy

Ions at Nanometer Site Sizes With a Wall-less Proportional

Counter

P. Kliauga

A Calculation of Exciton Energies in Periodic Systems with

Helical Symmetry: Application to a Hydrogen Fluoride Chain

M. G. Vracko and M. Zaider

Electron Energy-Loss Function in Polynucleotide and the

Question of Plasmon Excitation

A. Fung and M.Zaider

BIOPHYSICAL MODELLING

A Non-Parametric, Microdosimetric-Based Approach to the

Evaluation of the Biological Effects of Low Doses of Ionizing

Radiation

M. Zaider and M. Varma

High-LET Radiation Risk Assessment at Medium Doses

D. J. Brenner

High-LET Radiobiological Effects: Increased Lesion Severity

or Increased Lesion Proximity?

D. J. Brenner (In Collaboration with R. K. Sachs, Physics Department, University of California, Berkeley). 
Photoneutrons Generated by High Energy Medical Linacs

E. j. Hall

The Biological Effectiveness of Neutrons; Implications for

Radiation Protection

E. J. Hall and D. J. Brenner

Molecular Characterization of Oncogenes Induced by Neutrons

G. A. Freyer, Y. Yu and J. Feinstein

The Inverse Dose-Rate Effect for Oncogenic Transformation

by Charged Particles is LET Dependent

R. C. Miller, G. Randers-Pehrson, L. Hieber, S. A. Marino,

M. Richards and E. J. Hall

PUBLICATIONS 


\section{INTRODUCTION STAFF NEWS COLLOQUIA STAFF LIST TEACHING}




\section{INTRODUCTION}

The Center for Radiological Research of Columbia University epitomizes the multidisciplinary approach towards understan ling, at both basic and applied level, the biological and health problems related to human exposures to ionizing radiation. The strength of the Laboratory derives from its unique mixture of professionals in fields as diverse as molecular biology, physics, quantum chemistry, computational physics, radiation biology, biomathematics, experimental microdosimetry, and clinical radiation therapy. More often than not, answers to questions in any of these fields are just a short walk away on the corridors of the Center. The synergistic interaction among researchers is reflected in the scientific output of the Laboratory, both quantitatively and qualitatively. Last but not least, the availability of an inhouse DOE-sponsored accelerator facility (RARAF) significantly enhances our ability to perform quickly and efficiently most of the experimental work.

The Center is reshaping itself along new lines of research: Along with the more traditional fields of microdosimetry, cytogenetics and biophysical modeling (which have had a long tradition here) we now have groups in molecular biology and computational and theoretical physics. Both of these groups have attained critical mass in terms of scientific and support personnel; they are expected to play an increasingly leading role in this Laboratory's major research activities.

The following is a brief summary of research accomplishments in the Center. Further details may be found in the main text of this document and/or in the original literature quoted.

A) The Pulsed Brachytherapy concept, in which low dose rate brachytherapy is replaced with pulses of radiation, typically ten minutes in every hour, is now in clinical use. It is based on an idea derived and under continual development at the Center, and for which support has been obtained from Industry as well as from the National Cancer Institute.

B) Major components of our research activities involve oncogenic transformation assays. In a collaboration with the Medical Research Council in the U.K., we have investigated structure activity relationships for the potential oncogenicity of a new generation of bifunctional bioreductive drugs. A study is in progress of the biological effectiveness of photo - neutrons produced in high energy medical Linacs. Using transformation as an endpoint, misoprostal has been shown to be a good radiation protector when administered to animals in vivo.

C) The Monte Carlo code which transports electrons in condensed water is now functional. Other studies include calculations of exciton specific in poly cytosine and ab-initio estimation of the energy loss function of a DNA model. We continue the study of indirect effects of radiation on aqueous solutions of DNA. 
D) In collaboration with Dr. M.N. Varma of DOE and Dr. Wu of the Department of Radiation Oncology we are obtaining libraries of hit size effectiveness functions; these provide a direct and sensitive link between the microdosimetric description of the radiation field and dose equivalent factors, and are expected to play a major role in radiation protection.

E) In experimental nanodosimetry further evidence was accumulated that measurement of ionization distributions at the $10-20 \mathrm{~nm}$ level is possible and practicable with a miniature proportional counter. Work has begun on construction of a "wall-less" version.

F) Experimental and theoretical investigation of the "inverse dose rate effect", whereby medium LET radiations actually produce an enhanced effect when the dose is protracted, is now at a point where the basic mechanisms are reasonably understood - and the complex interplay between dose, dose rate and radiation quality which is necessary for the effect to be present can now be predicted.

G) Studies are continuing on the molecular characterization of radiation induced oncogenes. This work, using C3H 10T1/2 cells, has expanded into studies of neutron activated oncogenes. A cosmid library made from a neutron transformed cell line is currently being screened.

The productivity of the Laboratory continues at a high level, as evidenced by the steady stream of papers to peer-reviewed journals during the year, and invitations to members of the staff to participate in national and international meetings. Senior faculty are frequently called upon as consultants, reviewers and site visitors by government and private bodies.

Dr. Hall continues to serve on the BRER (Biological Research on the effects of Radiation) Committee of the National Academy of Sciences and the executive committee of the American Radium Society, while Dr. Geard serves on the American Cancer society Study Section in Prevention Diagnosis and Therapy.

Dr. Zaider has been invited to serve as a member of the "Expert Group on Hit Size Effectiveness Approach; Implications for Radiation Protection". He has also become a member of the Scientific Committee 88 of NCRP concerning the subject "Fluence as a Basis of a System of Radiation Protection for Astronauts".

Funding levels have been steady during the past year. Drs. Hei and Brenner successfully competed for awards from the National Cancer Institute and the American Cancer Society. 


\section{STAFF NEWS}

Dr. Laurie Roizin-Towle, Assistant Professor of Radiation Oncology left to take up a position in private industry after being associated with the Center for many years.

Dr. Zhu joined the laboratory as a post-doctorate fellow to work on problems of mutagenesis and transformation.

Ms. Louise Gurguis the Administrator for the Center affairs left in November 1991 to take up a new position as Administrator in the department of Anatomy and Cell biology. She was with us for over 5 years; we will miss her and wish her well in her new job. We welcome Ms. Monique Rey as the new Center Administrator.

Dr. Eric Hall gave the 1992 Janeway Lecture of the American Radium Society at Orlando, Florida and the Silvanus Thompson Memorial Lecture of the British Institute of Radiology at their annual meeting held at Birmingham, U.K.

Dr. David Brenner won the Robert Moseley Award of the National Council of Radiological Protection and Measurements. 


\section{THE COLUMBIA COLLOQUIUM AND LABORATORY SEMINARS}

At intervals of approximately one month during the academic year, a regular colloquium has been held to discuss ongoing research. Dr. Greg Freyer organized them and scheduled the speakers. These have been attended by the professional staff, graduate students, and senior technical staff of this Laboratory and RARAF, as well as scientists from other departments of the College of Physicians \& Surgeons interested in collaborative research. Attention has focused on recent findings and future plans, with special emphasis on the on the inter-disciplinary nature of our research effort.

During the year, we have been pleased to welcome a number of visitors who have presented formal seminars and/or spent time discussing ongoing research with various members of the Laboratory. These have included Dr. Johng S. Rhim, National Cancer Institute, Bethesda, MD, Dr. John F. Ward, University of California, San Diego, Professor G.E. Adams, United Kingdom, G. W. Barendsen, the Netherlands, Dr. James C. Willey, University of Rochester School of Medicine, New York, Dr. Chun-Zhang Chen, University of California, San Francisco, Dr. Yutaka Okumura, Nagasaki, Japan and Dr. Marian Cholewa, University of Melbourne, Australia. 


\section{CENTER FOR RADIOLOGICAL RESEARCH}

\section{RESEARCH STAFF}

* ERIC J. HALL, D. Phil, D.Sc, FACR, Professor of Radiology and Radiation Oncology (Director)

* CHARLES R. GEARD, Ph.D., Professor of Clinical Radiation Oncology (Associate Director)

* DAVID J. BRENNER, Ph.D., Assistant Professor of Radiation Oncology DIANE ESPOSITO, Ph.D., Associate Research Scientist (until Dec. 1991)

* GREG A. FREYER, Ph.D., Assistant Professor of Radiation Oncology ZHU-YING HE, M.D., Staff Associate TOM K. HEI, Ph.D., Assistant Professor of Radiation Oncology KEVIN HOPKINS, M.S., Staff Associate

* PAUl J. KLIAUGA, Ph.D., Assistant Professor of Radiation Oncology HOWARD B. LIEBERMAN, Ph.D., Assistant Professor of Radiation Oncology BRADFORD LOUCAS, Ph.D., Associate Research Scientist

* STEPHEN A. MARINO, M.S., Senior Staff Associate RICHARD C. MILlER, Ph.D., Assistant Professor of Clinical Radiation Oncology

CHANG-QING PIAO, M.D., Senior Staff Associate

* GERHARD RANDERS-PEHRSON, Ph.D., Associate Research Scientist LAURIE ROIZIN-TOWLE, Ph.D., Assistant Professor of Radiation Oncology (until November 1991)

* MARCO ZAIDER, Ph.D., Associate Professor of Radiation Oncology and Public Health 


\section{POST-DOCTORAL FELLOWS}

- MARJAN VRACKO-GROBELSEK, Ph.D., Post-doctoral Research Scientist KHALIFAH SIDIK, Ph.D., Post-doctoral Research Scientiest

- $\quad$ YONG YU, M.D., Staff Associate

YUQI ZHAO, Ph.D., Post-doctoral Research Scientist

LI XUN ZHU, M.D., Post-doctoral Research Scientist

* Partially or wholly supported by the Department of Energy 


\section{DESIGN AND INSTRUMENT SHOP}

* GARY W. JOHINSON, A.A.S., Senior Staff Associate (Director)

* NICHOLAS LUDAS, Senior Research Worker

* DAVE CASSIMY, Instrument Maker

\section{TECHNICAL STAFF}

JULIE FEINSTEIN, M.A., Senior Technician

JOSE GARCIA, Laboratory Assistant

MARCUS GEARD, B.A., Technician B

GLORIA JENKINS, B.A., Research Worker

JACQUELINE JONES, B.A., Technician B

* JESUS PEREZ, Accelerator Technician

* MARCIA RICHARDS, B.S., Research Worker

\section{ADMINISTRATIVE AND SECRETARIAL STAFF}

* MONIQUE REY, B.A., Center Administrator (from Nov. 1991)

* LOUISE C.T. GUIRGUIS, M.S., Center Administrator (until Oct. 1991)

* MARIE BURCHETT, Senior Secretary YVETTE CLAUSELL, A.A., Execuiive Secretary

* MARY COADY, Administrative Assistant KAREN EVANS, B.A., Junior Accountant C. JEANNE KRAMER, Senior Secretary 


\section{TEACHING}

Dr. Eric Hall taught in the Oncology Section of the course on "Abnormal Human Biology" for second-year medical students. In addition, a laboratory elective for medical students in "Cellular and Radiation Biology" was offered by Drs. Hall and Geard.

Drs. Brenner and Lieberman taught a course, "Radiation and Life" for non-science undergraduates, under the auspices of the Department of Biological Sciences.

Dr. Zaider is the Director of the Graduate Program in Medical Physics/Health. Physics, offered jointly vy the School of Public Health and the College of Physicians \& Surgeons. Dr. Zaider is also supervising the Ph.D. work of Albert Fung of the Physics Department, Columbia University.

During the Fall, a didactic course in Radiobiology was given by Drs. Hall, Geard, Brenner, Freyer, Hei, Miller, and Lieberman, for Columbia Presbyterian residents in Radiation Oncology and other institutions in the New York area. Drs. Hall and Geard also taught a course in Radiobiology for residents in Diagnostic Radiology. A similar course was taught at Montefiore Hospital, as well as a one-day refresher course. Drs. Geard and Miller lectured to Radiological Technologists at United Hospitals, Port Chester, NY, and delivered update lectures to Radiological Technologists in Presbyterian Hospital. Drs. Astor, Towle, and Hall also participated in the teaching of a Radiobiology course for residents in the Department of Radiation Therapy at the University of Pennsylvania.

Dr. Kliauga taught a laboratory course, "Nuclear Instrumentation." Drs. Zaider and Brenner are lecturing on radiation effects in the courses entitled "Industrial Toxicology" and "Environmental Sciences," while Dr. Geard is lecturing in the course "Environmental and Molecular Toxicology." Additionally, Dr. Zaider is conducting a laboratory on the detection, measurement, and risk evaluation of radon in housing environments.

Dr. Greg Freyer teaches in the Medical Genetics at Columbia University. He is also a member of the Summer High School Science Teacher Program, and hosts a high school teacher, in his laboratory. 


\section{RADIATION PHYSICS AND MICRODOSIMETRY}




\title{
Design and DeVelopment of a NeW WALL-Less UltaA Miniature PROPORTIONAL COUNTER FOR NANODOSIMETTYY
}

\author{
Paul Kllauga and Gary Johnson
}

\section{Introduction}

During this past year, experimental work on nanodosimetry has concentrated on the design and construction of a new wall-less proportional counter based on the "ultra miniature" design developed in earlier walled types ${ }^{1,2}$. The design follows a prototype which was constructed for heavy ion microdosimetry at the Brookhaven Tandem Van de Graaf and at RARAF. Some recent results with that counter operated at low pressures to simulate nanodosimetric site sizes are summarized in another paper in this report ${ }^{3}$.

Those results provided very convincing evidence that a wall-less counter of small dimensions is able to provide experimental microdosimetric information on ionization distributions in tissue equivalent volumes down to about $20 \mathrm{~nm}$ with the prototype counter. It is expected that the counter currently under construction will achieve acceptable gain and resolution down to at least $10 \mathrm{~nm}$.

The basic design considerations for such a counter include:

- A simple cylindrical geometry, which makes miniaturization practicable, and provides the large electric field strengths needed near the center wire.

- Careful selection of materials. Insulators must have good dielectric strength, excellent degassing properties and resistance to radiation damage. The helix wire material must be chosen with the proper tensile strength and hardness to be able to provide a uniform "spring" when wound into a coil with dimensions of the order of $1 \mathrm{~mm}$.

- Extreme care in construction tolerances. Alignment and symmetry is critical to produce acceptable resolution in a counter of this size. Therefore all parts are custom made to fit to as near zero tolerance as possible. The plastic (Rexolite) collars to hold the field tube assemblies in alignment in the aluminum yoke are very critical parts which have been machined (see Fig.2) to provide alignment tolerance of about $0.003 \mathrm{~mm}$ at the center of the counter.

\section{Design of the Counter}

One of the major concerns is field uniformity and symmetry. This influences the operational characteristics of the counter more than any other factor. It was essential

\footnotetext{
${ }^{1}$ Kliauga, Paul, Measurement of Single Event Energy Deposition Spectra at $5 \mathrm{~nm}$ to $250 \mathrm{~nm}$ Simulated Site Sizes, Rad. Prot. Dos. 31, 119-123 (1990)

${ }^{2}$ Kliauga, Paul, Microdosimetry at Middle Age: Some Old Experimental Problems and New Aspirations, Rad. Res. 124, S5-S15 (1990)

${ }^{3}$ Kliauga, Paul Some Recent Measurements of Ionization Distributions for Heavy lons at Nanometer Site Sizes With a Wall-less Proportional Counter, This Report
} 
to know just how far insulating pieces, for example, had to be placed from the collecting volume in order to produce negligible distortion of the field in the critical ion collection region. In order to investigate this we have plotted solutions to the two dimensional Poisson equation for a simple cylindrical geometry with insulator pieces of various sizes and geometrical positions in order to determine an optimum design of those pieces which hold the field tubes and provide insulation as well as give mechanical strength to the field tube assembly. Using such a plot it is also possible to determine optimum ratio of helix/center wire potential to produce the best cylindrical symmetry in the collecting volume. An example is shown in Fig. 1.

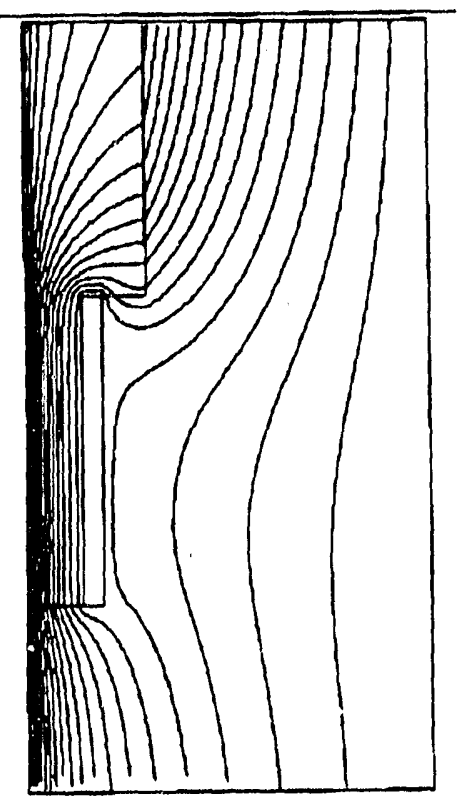

Fig. 1a. $V_{C W=+400 ;} V_{H}=-100$

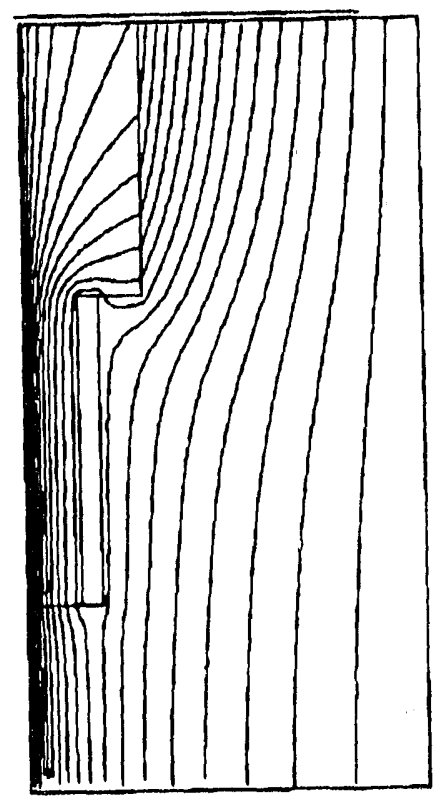

Fig. 1b. $W_{C W=+300 ; V_{H}=-200}$

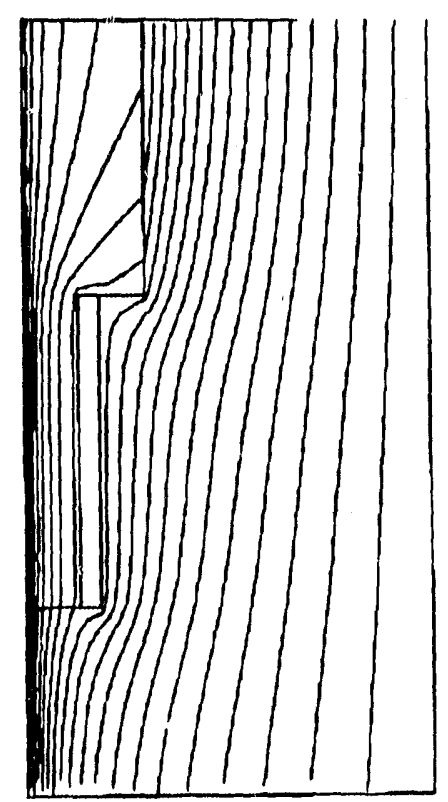

Fig. 1c. $V_{C W}=+200 ; V_{H}=-300$

Figure 1. Solutions to the two-dimensional Poisson equation for various ratios of helix/center wire potentials. The total potential difference is constant, only relative values change. The field rube is at ground potential. Fig. 1b $(\mathrm{VCW}=+300 ; \mathrm{VH}=-200)$ represents the best case, in which the equipotentials are radially symmetric.

These figures are plots of equipotential lines in the vicinity of the filed tube and canter wire. The helix is at a larger radius than shown here. The plots show the distortion of the equipotential lines near the insulators as well as the effect of varying the ratios of potentials on the conducting surfaces.

The counter design is shown in Figure 2. The yoke is constructed of specially stressannealed aluminum to reduce distortion due to machining. This piece required a great deal of time and several attempts to attain the degree of precision required for accurate alignment of the two opposite field tubes. Plastic parts of the field tube assembly are currently being machined. The entire counter assembly will be placed inside of an enclosure large enough to assure adequate "wall-lessness" and mounted on a precision xyz motion so that the counter can be moved relative to a fixed source. 

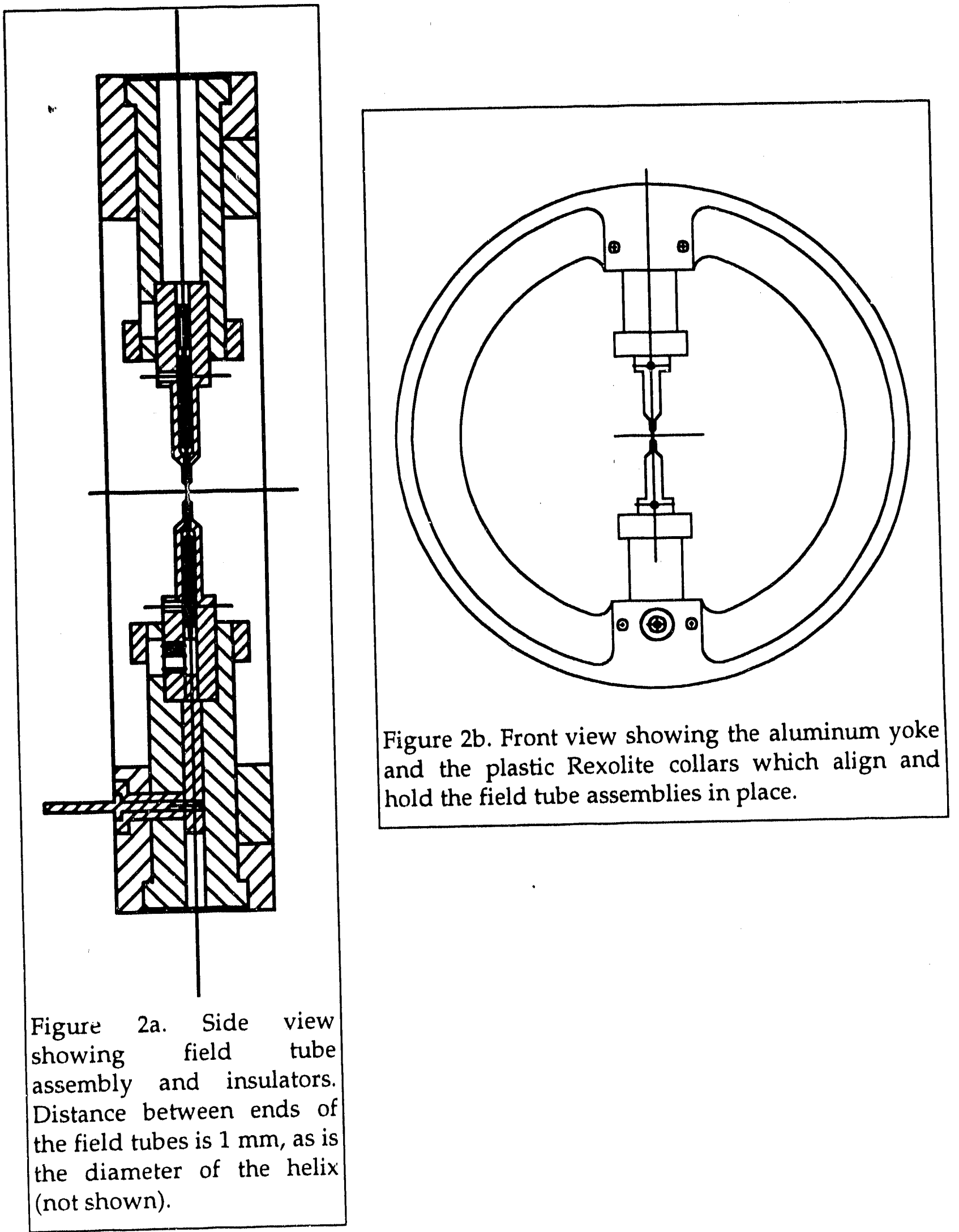


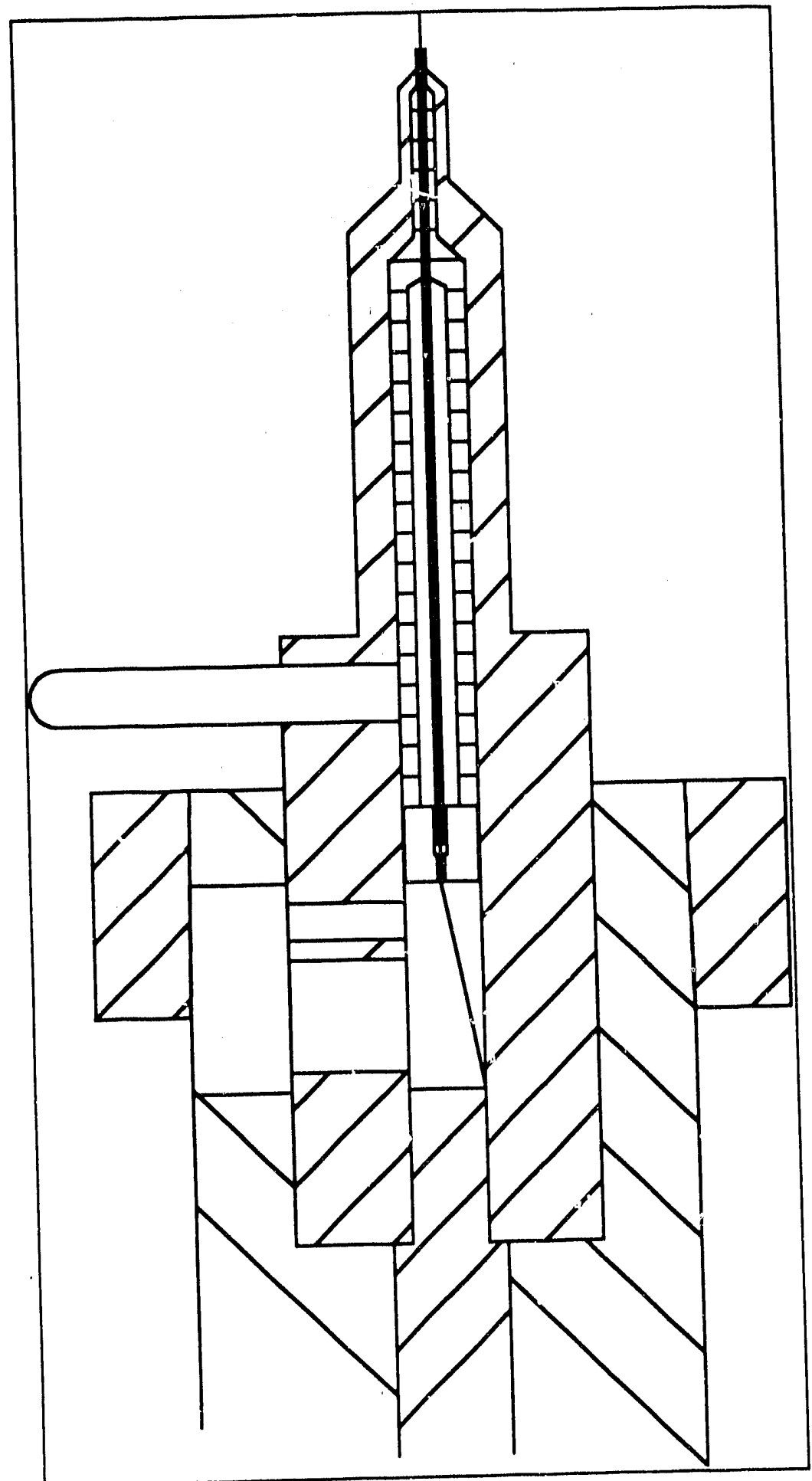

Figure 3. Closeup of the field tube assembly. The microbore glass tube which centers the anode and provides insulation is shown, with the center wire protruding from it. The I.D. of the glass tube is 15 $\mathrm{mm}$ the O.D. is $150 \mathrm{~mm}$. The horizontal cross-hatched area is the aluminum sleeve which is machined down to a wall thickness of $25 \mathrm{~mm}$ to create a field tube in the region where the glass tube emerges from the insulating support into the active collecting region. The helix (not shown) is supported on the same Rexolite piece which supports the field tube/glass tube assembly. 


\title{
SOME RECENT MEASUREMENTS OF IONIZATION DISTRIBUTIONS FOR HEAVY IONS AT NANOMETER SITE SIZES WITH A WALL-LESS PROPORTIONAL COUNTER
}

\author{
Paul Kliauga
}

\section{Introduction}

About two years ago we had constructed a new wall-less cylindrical counter designed for heavy ion work at the Brookhaven Tandem van de Graaf and at RARAF. The counter was deliberately designed with small dimensions so that it would be possible to attain radial delta ray equilibrium with a beam of nominal size (about $6 \mathrm{~mm}$ diameter) for the heavy ions of interest. A size of $3.175 \mathrm{~mm}$ was chosen $\left(1 / 8^{\prime \prime}\right)$. Attempts to operate this counter at reduced pressures to simulate nanometer size tissue equivalent sites provided encouraging results. Some initial efforts were first reported in last year's annual report, and subsequently published. We report here some additional evidence, including a comparison with a simple theoretical model to show that the counter does indeed produce useful spectra down to 20 nanometers.

\section{Experimental}

A few years ago a wall-less counter was constructed specifically for use with charged particle beams. This was a part of the so-called "Q Project", another part of this research program. The counter is a right regular cylindrical counter with the counting volume defined by a wire helix along the diameter and by field tubes along the axis. The dimensions so defined are $3.175 \mathrm{~mm} \times 3.175 \mathrm{~mm}$. Thus, while larger than the UMC, it is still quite small compared to conventional microdosimetric proportional counters. These dimensions were deliberately chosen so as to provide radial delta ray equilibrium with a $6.4 \mathrm{~mm}$ beam spot for the majority of the charged particle beams used at Brookhaven national Laboratory's Tandem Van de Graaf and at RARAF. The entire counter is fixed on an aluminum ring and mounted inside of a brass cylindrical container holding the tissue equivalent gas. Entrance to the counting volume for the charged particle beams is through a $6 \mu \mathrm{m}$ mylar window. The window plus the depth of the gas between the window and the center of the counter in equivalent unit density tissue is supposed to simulate a cell plated on $6 \mu \mathrm{m}$ mylar dish and irradiated through the mylar.

Charged particles provide an excellent source for testing counter operation. A monoenergetic beam whose energy is large compared to the energy loss in the counter provides a constant LET source whose energy deposit in the counter is determined entirely by ionization statistics and the chord length distribution in the counter. The resultant $y$-distributions are predictable and simple. Therefore, even though the " $Q$ " counter is considerably larger than the UMC, it was attempted to operate it at pressures low enough to simulate site sizes down to about $10 \mathrm{~nm}$. 
Because of the large size of the containing volume, pressure regulation was not difficult even at only about 1 Torr.

Two beams were chosen for these tests, one high LET beam, $110 \mathrm{MeV} \mathrm{O}^{+}$, with an LET of approximately $380 \mathrm{keV} / \mu \mathrm{m}$, and a low LET $16 \mathrm{MeV} \mathrm{d}+$ beam having an LET

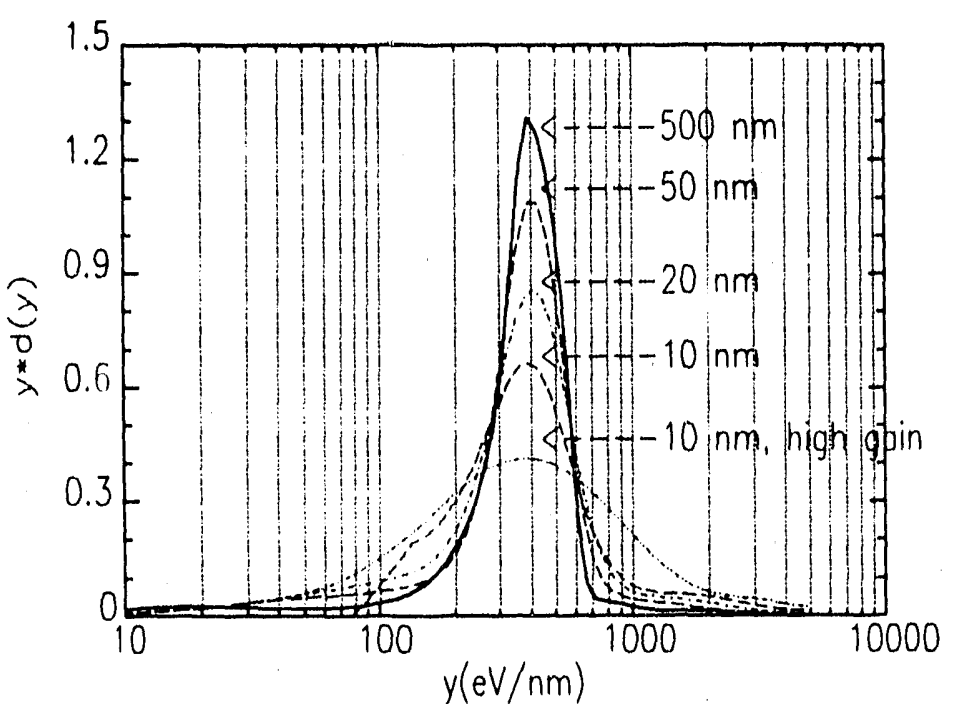

Figure 1a. Dose distributions for $110 \mathrm{MeV}$ oxygen ions with LET of approximately $380 \mathrm{keV} / \mathrm{mm}$.

of approximately $6 \mathrm{keV} / \mu \mathrm{m}$. The dose and frequency distributions in lineal energy for the oxygen beam are shown in Figs. 1a and $1 \mathrm{~b}$.

Two things should be noted about these distributions. 1) In the dose distributions, resolution of the counter is maintained down to $20 \mathrm{~nm}$. Only at $10 \mathrm{~nm}$ does the spectrum become noticeably wider than would be accounted for simply by statistics. Even at $10 \mathrm{~nm}$, provided the gain is not made too large, the widening is not unacceptable. At larger gain, noticeable loss of resolution occurs for $10 \mathrm{~nm}$. 2) In the frequency distributions, the low LET peak which is not readily visible on the dose distributions becomes prominent. These "delta ray peaks" are ohserved in all wallless spectra of charged particle beams. These peaks indicate that a major component is slow electrons (stoppers) injected into the counting volume from particles passing near but not through the counting volume, because the peaks shift to the right with decreasing site size. The other beam used in these

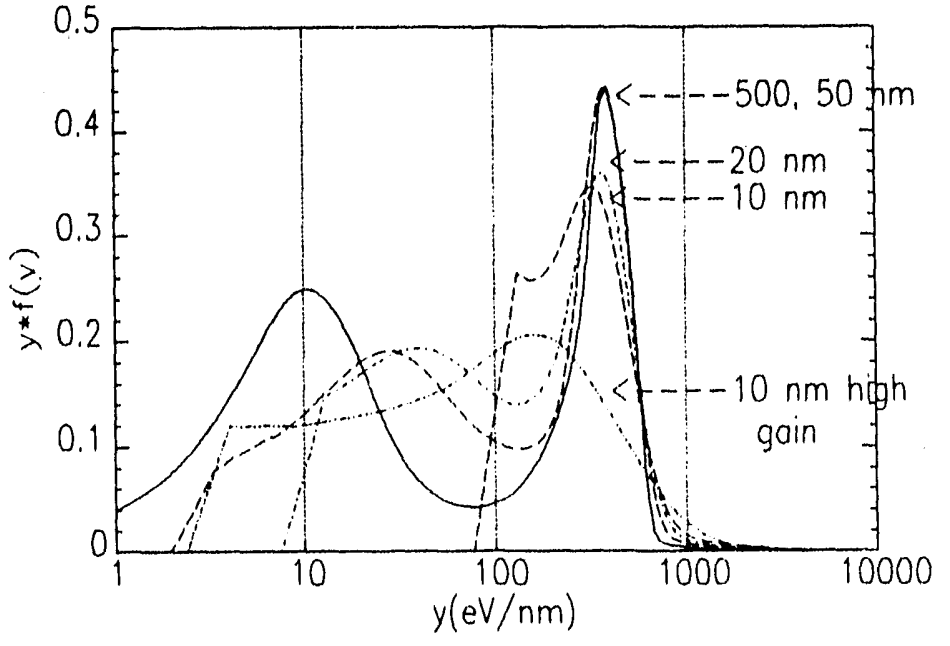

Figure 1b. Frequency distributions for $100 \mathrm{MeV}$ oxygen ions. Note "delta ray" peaks. tests is a low LET deuteron beam. Dose distribution in lineal energy is shown for a $20 \mathrm{~nm}$ site size in Fig. 2. The mean chord length for a parallel beam incident on a cylinder perpendicular to its axis is just the mean chord length of a circle, $d / 2$. Thus for a $20 \mathrm{~nm}$ diameter, the mean chord length is $10 \mathrm{~nm}$ for this geometry. At 6 $\mathrm{eV} / \mathrm{nm}$, and assuming $\mathrm{W}$ about $30 \mathrm{eV}$ per ion pair, this corresponds to about 2 ionizations on the average. Of course, there will be a distribution of ionizations, 
determined by both the Poissonian distribution of ionization probability and the distribution of chord lengths. As in the case of the UMC, we can simulate a single ion event by assuming an exponential distribution of pulse heights and converting this to a microdosimetric dose distributior. From this single ion distribution it is easy to fold these together to create $2,3,4$, itc. ion distributions. Such distributions are noted in Figure 2 with the number of ions next to the peak. These distributions can be combined with appropriate weighting factors to produce the composite spectrum. In our case, the weighting factors have been estimated on the basis of chord length distributions. It is intended to obtain more accurate weight factors from Monte Carlo simulations of such a geometry. The measured spectrum matches the theoretical one quite well in shape. Its variance, or FWHM, is about $20 \%$ larger than the theoretical.

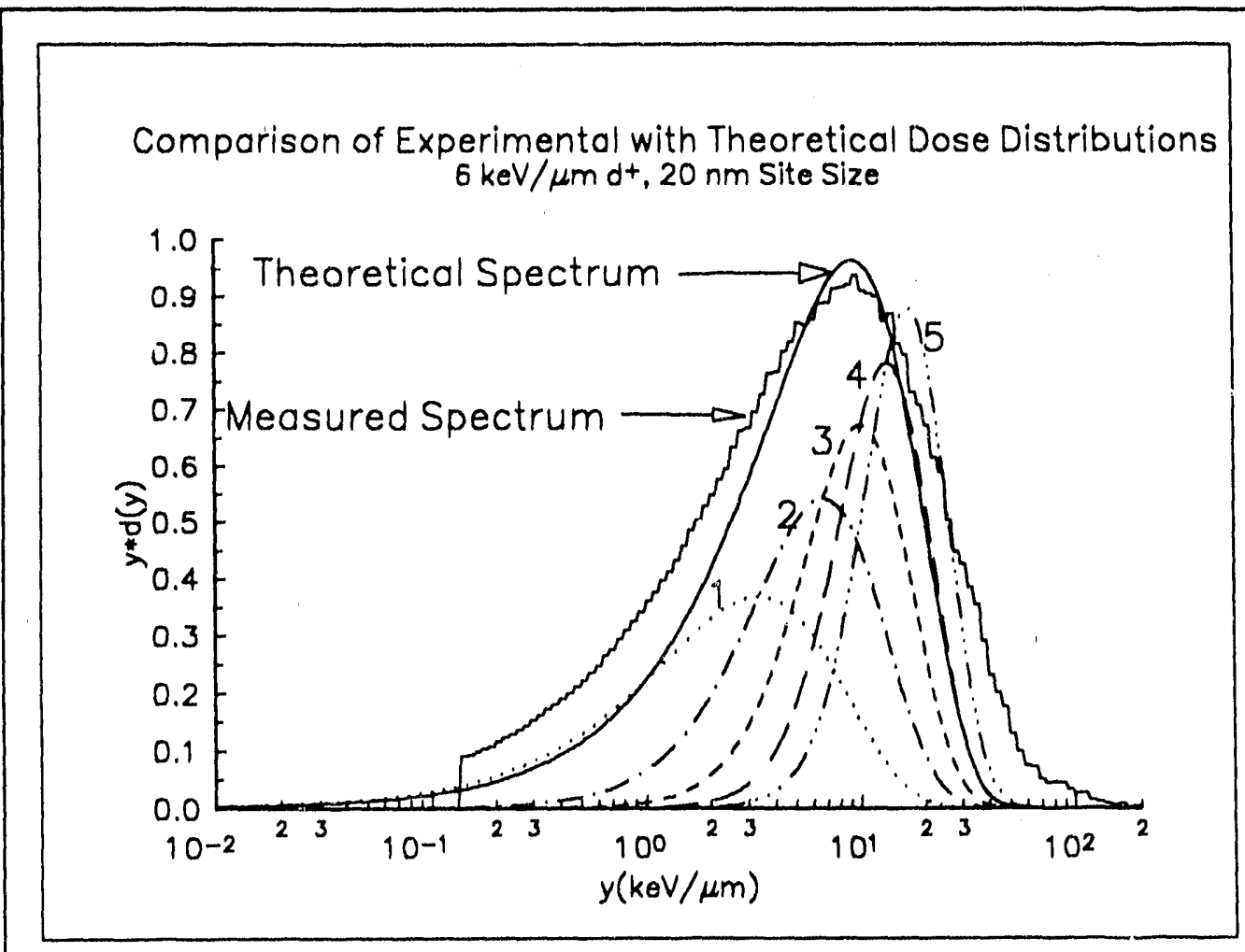

Figure 2. Dose distribution for $6 \mathrm{keV} / \mathrm{mm}$ deuterons at $20 \mathrm{~nm}$ site size. compared to a "theoretical" distribution obtained by folding theoretical single-event distributions.

\section{Summary}

These data provide quite convincing evidence that not only the UMC but the wallless " $Q$ " counter is capable of measurements at the nanometer level. With a slight reduction in resolution the $Q$ counter produces accurate and predictable spectra down to $20 \mathrm{~nm}$ which agree within acceptable limits with theoretical prediction. The UMC appears able to do so to at least $10 \mathrm{~nm}$. It has been demonstrated that we now possess instruments which are able for the first time to measure energy (ionization) deposition spectra at the nanometer level. 


\title{
A Calculation of Exciton Energies in Periodic Systems with Helical SYMMETAY: APPLICATION TO A HYDROGEN FLUORIDE CHAIN
}

\author{
M. G. Vracko and M. Zaider
}

\section{Introduction}

Because of the role they play in biochemistry, polymers with helical symmetry have been the subject of many theoretical studies. These systems have been treated up to the Hartree-Fock approximation and it was shown, that they can be described in the same way as systems with translation symmetry only: the eigenvalues and eigenfunctions of the Hamiltonian (or Hartree-Fock operator) may be classified according to the band index and quasi-momentum in the first Brillouin's zone. This latter corresponds to a combined translation $\tau$ and rotation $\phi$ (we assume $n \phi=2 \pi$, where $n$ is an integer). In this article we present an extension of the Hartree-Fock approximation to the calculation of exciton energies. The excitons have been treated within the intermediate exciton theory (see Methods). As an example, we apply these methods to the hydrogen fluoride (HF) polymer.

A considerable number of calculations have been performed using the HF chain as a model for solid HF. Solid HF consists of (HF) units build up as one-dimensional chains with large interchain distances. Therefore, it should be justified to treat it as a one-dimensional system. Its electron structure has been calculated in the tight binding and Hartree-Fock approximation. Correlations calculation have been performed for different clusters with the Moller-Plesset perturbation theory up to the second order. In the quasi-particle band structure (up to the third order in the Moller-Plesset perturbation theory) and the exciton energies up to the second order were reported. Since for this system $n=2$, two HF units were chosen as elementary cell, thus making the system translationally invariant. The effect of helical symmetry has been investigated in in the Hartree-Fock approximation.

\section{Mothods}

In a number of studies the excitons have been treated in the framework of the intermediate exciton theory. An attempt has been made to find a general formulation for excitons which are neither of Frenkel type (i.e. a pair of a localized electron and hole) nor of Wannier type (i.e. a nonlocalized electron-hole pair).

Typically the exciton wave function has been chosen as a linear combination of Slater determinants in which one Bloch function of the occupied band (with quasimomentum $\mathrm{k}$ ) is replaced with one of the unoccupied bands with quasi-momentum $\mathrm{k}+\mathrm{K}$, where $\mathrm{K}$ is to the exciton momentum. In several reports the Bloch functions are expressed in terms of Wannier functions in order to compare this method with the Frenkel formulation, or to evaluate general features (e.g. the efficiency of the calculation).

In this calculation we have used the method of two-particle (particle-hole) timedependent Green's functions in the Tamm-Dancoff (TD) and the random-phase (RP) 
approximation. In these approximations the equation for the particle-hole propagator has been rewritten for periodic systems with first order irreducible vertex parts. In the TD approximation the forward time ordering in the expression for the interaction-free particle-hole propagator has been used. This corresponds to the method mentioned above. In Eq. (1) (see below) the interaction-free particle-hole propagator $\left(G_{\alpha \beta}^{(0)-1}(\omega)\right)$ describing the movement of particle-hole pair forward in time is given in the $\omega$ representation. It is coupled to the irreducible vertex part defined in the expression (4) below:

$$
\left\{G_{\alpha \beta}^{(0)-1}(\omega) c_{\alpha \beta}^{(1)}-\sum_{\lambda \mu} K_{\alpha \beta \lambda \mu}^{(1)} c_{\lambda \mu}^{(1)}\right\}=0
$$

In the RP approximation both time orderings, the forward and the backward, have been taken into account. In Eq. (2) both propagators are given in the $\omega$ representation $\left(G_{\alpha \beta}^{(0)-1}(\omega)\right.$ and $\left.G_{\alpha \beta}^{(0)-1}(-\omega)\right)$ and the subspaces belonging to the different time-orderings are coupled with a part of the irreducible vertex $K_{\alpha \beta \lambda \mu}^{(2)}$

$$
\begin{aligned}
& \left\{G_{\alpha \beta}^{(0)-1}(\omega) c_{\alpha \beta}^{(1)}-\sum_{\lambda \mu}\left(K_{\alpha \beta \lambda \mu}^{(1)} c_{\lambda \mu}^{(1)}+K_{\alpha \beta \lambda \mu}^{(2)} c_{\lambda \mu}^{(2)}\right)\right\}=0 \\
& \left\{G_{\alpha \beta}^{(0)-1}(-\omega) c_{\alpha \beta}^{(2)}-\sum_{\lambda \mu}\left(K_{\alpha \beta \lambda \mu}^{(1)} c c_{\lambda \mu}^{(2)}+K_{\alpha \beta \lambda \mu}^{(2)} c_{\lambda \mu}^{(1)}\right)\right\}=0
\end{aligned}
$$

where $\alpha, \beta, \lambda, \mu$ are combinations of band and quasi-momentum indices (according to the expression (4) $\alpha, \mu$ are related to the conduction and $\beta, \lambda$ to the valence band index). Furthermore, the $c \stackrel{(1)(2)}{\lambda \mu}$ are the eigenvectors of the Eq. $(1,2)$. The explicit expressions for $G_{\alpha \beta}^{(0)-1}(\omega)$ and $K_{\alpha \beta \lambda \mu}$ are:

$$
\begin{gathered}
G_{\alpha \beta}^{(0)-1}(\omega)=\omega-\varepsilon_{k_{i} c}+\varepsilon_{k_{i}+K v} \\
K_{\alpha \beta \lambda \mu}^{(1)}=-\left\langle k_{i} c, k_{i}+K v\right| k_{j} c^{\prime}, k_{j}+K v^{\prime}>+ \\
g^{*}<k_{i} c, k_{i}+K v \mid k_{j}+K v^{\prime}, k_{j} c^{\prime}> \\
K_{\alpha \beta \lambda \mu}^{(2)}=-<k_{i} c, k_{i}+K c^{\prime} \mid k_{j} v^{\prime} k_{j}+K v>+ \\
g^{*}<k_{i} c, k_{i}+K c^{\prime} \mid k_{j}+K v, k_{j} v^{\prime}>
\end{gathered}
$$

$\mathrm{g}$ is 0 for triplet and 2 for singlet excitons and $c, v, c^{\prime}, v^{\prime}$ are indices for the conduction and valence band, respectively. For a discrete grid of $k$ points the $G_{\alpha \beta}^{(0)-1}(\omega)$ and 
$\mathrm{K}_{\alpha \beta \lambda \mu}$ in Eqs. $(1,2)$ must be multiplied with the appropriate weights for numerical integrations and they become matrix equations. Furthermore, we used the following notation for the two-electron integrals:

$\left\langle k_{i} n_{1}, k_{j} n_{j} \mid k_{m} n_{m}, k_{n} n_{n}\right\rangle=\iint d r_{1} d r_{2} \frac{\phi_{k_{j} m_{1}}^{*}(1) \phi_{k_{j} n_{j}}^{*}(2) \phi_{k_{m} n_{m}}(1) \phi_{k_{n} n_{n}}(2)}{\left|r_{1}-r_{2}\right|}$

where $\phi_{k n}$ are Hartree-Fock orbitals.

The Eqs. $(1,2)$ have several possible solutions i.e., for chosen exciton quasimomentum $\mathrm{K}$ and band indices there is a continuum spectrum of exciton energies (if the number of $k$ values in Eqs.(1-4) tends to infinity). We investigated just the few lowest energies lying in the Hartree-Fock energy gap.

\section{Computational Details and Results}

The program IBMOL was used to solve the Hartree-Fock equation. It was solved at $7 \mathrm{k}$ points (in the irreducible part of the first Brillouin's zone) and by considering two next-neighbor interactions. The two-electron integrals for the calculation of the irreducible vertex part were calculated in the single-neighbor approximation. Eqs. $(1,2)$ were solved at the exciton momentum $K=0$ and $K=\pi$ to evaluate the width of exciton bands.

Furthermore, the Eqs. $(1,2)$ have been solved twice, first without the band-band interaction setting $c=c^{\prime}$ and $v=v^{\prime}$ in the expression (4) and second taking the band-

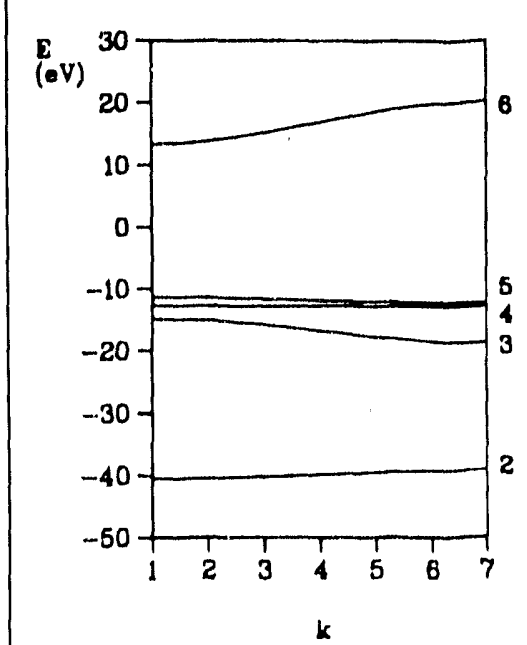

Fig. 1: The Hartree-Fock band structure of the helical HF chain in the irreducible part of the first Brillouin's zone. (Calculated with the STO-3G basis.) band interaction into account.

The calculations were performed using the STO-3G.

The experimental geometry was taken from, i.e. $1.02 \AA$ for the $\mathrm{H}-\mathrm{F}$ and $2.49 \mathrm{~A}$ for the F-F bondlength distance. The F-F-F angle was $120.1^{0}$. One H-F unit was chosen as the elementary cell, and the helical angle was taken as $180^{\circ}$.

The Hartree-Fock band structure in the valence region is shown in Fig.1. Bands 2.5 are occupied. The core band is lying at $-704 \mathrm{eV}$ and is omitted from the figure. The Hartree-Fock eigenvectors of the bands $2,3,5$ and 6 are the combinations of $\mathrm{s}, \mathrm{py}$ and $\mathrm{p}_{\mathrm{z}}$ functions, i.e. the functions located in the $(\mathrm{H}-\mathrm{F}) \mathrm{n}$ plane ( $\sigma$-bands). The band 4 is of $\Pi$-symmetry, i.e. the eigenfunctions are combinations of the functions located perpendicular to the plane.

The exciton energies have been calculated using the 
bands 3-5 as valence and 6 as conduction band. The energies calculated from the bands 2 and 6 lie at $40 \mathrm{eV}$ and are far from the Hartree-Fock gap.The results are shown in the Tables 1a,b. It is apparent from these tables that the TD treatment gives results which are very close to the RP approximation; the differences in exciton energies are between 0 and $0.8 \mathrm{eV}$ (i.e. less than $5 \%$ ).

Table 1a: The exciton energies in the Tamm-Dancoff approach $(s=$ singlet, $t=$ triplet).

A : The lowest exciton energies calculated without the bandband interaction represent 3-5 valence bands and 6 the conduction band.

$B$ : The five lowest exciton energies calculated with the bandband interaction.

STO-3G results (Hartree-Fock gap is $24.636 \mathrm{eV}$ )

A

B

\begin{tabular}{|c|c|c|c|c|c|c|c|c|}
\hline$c / v$ & $6 / 3$ & $6 / 4$ & $6 / 5$ & & & & & \\
\hline \multicolumn{9}{|l|}{$k=0$} \\
\hline $\mathbf{s}$ & 23.482 & 14.003 & 15.497 & 23.516 & 22.953 & 20.956 & 14.003 & 13.653 \\
\hline$t$ & 15.225 & 12.600 & 13.945 & 19.498 & 19.388 & 13.573 & 12.591 & 12.565 \\
\hline \multicolumn{9}{|l|}{$k=\Pi$} \\
\hline $\mathbf{s}$ & 23.837 & 13.468 & 14.030 & 22.521 & 20.248 & 19.901 & 13.464 & 12.815 \\
\hline$t$ & 15.136 & 12.085 & 12.543 & 23.337 & 21.899 & 14.267 & 12.080 & 11.671 \\
\hline
\end{tabular}

Table 1b: The exciton energies in the random-phase approach.

STO - 3G results

A

B

\begin{tabular}{|c|c|c|c|c|c|c|c|c|}
\hline $\mathrm{c} / \mathrm{v}$ & $6 / 3$ & $6 / 4$ & $6 / 5$ & & & & & \\
\hline \multicolumn{9}{|c|}{10717 12060 12626} \\
\hline $\mathbf{s}$ & 23.339 & 13.970 & 15.448 & 22.902 & 20.949 & 19.717 & 13.969 & 13.626 \\
\hline $\mathrm{t}$ & 14.201 & 12.589 & 13.857 & 19.535 & 19.337 & 13.455 & 12.589 & 11.989 \\
\hline \multicolumn{9}{|c|}{$k=\Pi$} \\
\hline $\mathbf{s}$ & 23.612 & 13.455 & 13.928 & 22.283 & 20.211 & 19.001 & 13.455 & 12.584 \\
\hline $\mathrm{t}$ & 14.341 & 12.075 & 12.479 & 19.534 & 19.462 & 14.000 & 12.071 & 11.547 \\
\hline
\end{tabular}


The calculations were performed with or without band-band interaction and the results are given in the Table 1a,b (denoted with $B$ and $A$ ). In the case $B$ the five lowest exciton energies are reported. It is obviously that the band-band interaction is most important between the $\sigma$ bands. The excitons $6 / 5$ (conduction/valence band) are strongly affected while the $6 / 4$ excitons are not affected by the band-band interaction. In the exciton calculation from the deeper valence region ( $6 / 3$ excitons) the band-band interaction becomes essential and the approximation $A$ breaks down.

\section{Conclusions}

The exciton bands for linear periodic structures with helical symmetry have been investigated. Calculations of excitons in the random-phase and the Tamm-Dancoff approximation have been compared. It was found the Tamm-Dancoff method is a very good approximation to the random-phase approach. We have also extended the previous approximation by taking into account the band-band interaction. This interaction plays an important role for bands of the same symmetry. These calculations were performed for a hydrogen fluoride polymer. Calculations on more complex systems (e.g.homopolynucleotides) are in progress in our laboratory. 


\section{Albert Fung and Marco Zaider}

In the first Born approximation, the energy deposition of an electron passing through condensed matter can be characterized by a single quantity, the dielectric response function, $\varepsilon$. Specifically, the electron inelastic differential cross section is proportional to $\operatorname{Im}(-1 / \varepsilon)$, called the energy-loss function. Experimental data are insufficient to understand fully the mechanism of energy deposition. Optical spectroscopy or electronic scattering only provide measurements at small momentum transíer $(q \approx 0)$. To obtain the energy loss for any momentum transfer as required in a Monte Carlo study, a theoretical approach must be sought. There exist theories to obtain the dielectric response function and energy-loss function, when the eigenfunctions and the energy bands of the substance are known. We use an expression based on the random-phase approximation to calculate the energy-loss function of polycytosine, a model polynucleotide.

The dielectric response function at momentum transfer $k$ and energy transfer $\hbar \omega$ can be found with (1):

$$
\varepsilon(q, \omega)=1+\frac{4 \pi e^{2}}{q^{2} \Omega} \sum_{n 1 k} \frac{k 1 k+q\left|e^{i q \cdot r}\right| n k>\left.\right|^{2}}{E_{1}(k+q)-E_{n}(k)-\hbar \omega+i \hbar \beta},
$$

where $E_{n}(k)$ is the energy of the state $\mid n k>$ at momentum $k, \Omega$ is the unit cell volume, and the summation is over all the occupied bands $n$ and unoccupied band 1. The local field effect, due to the rapid variation of microscopic electric field over the unit cell, is ignored. Since the wavefunction is continuous over $k$, the summation over $\mathbf{k}$ can be changed to an integral, and for a one-dimensional polymer is along one direction only. Thus we need to calculate an expression of the type:

$$
I(\omega)=\lim _{\beta \rightarrow 0} \int d k \frac{M(k)}{E(k)-\hbar \omega+i \hbar \beta},
$$

where $q$ is suppressed, $M(k)$ is the square of the matrix element, and $E(k)=E_{1}(k+q)$ $E_{n}(k)$. The real and imaginary parts are related together by the Kramers-Kronig formula. When $M(k)$ and $E(k)$ are known at points along the Brilluoin zone (BZ), interpolation can be performed on $E(k)$, so that one obtains closed-form expressions for $I(\omega)$.

We studied a simple polymer with screw symmetry, in which the nucleotides along the DNA helix are identical, and which remains unchanged in configuration after a combined translation and rotation about the helix axis. Polymers have onedimensional periodicity and therefore periodic electronic density: 


$$
\rho(\mathbf{r}+v \mathbf{a})=\rho(\mathbf{r}),
$$

where $a$ is the translational vector of a unit cell (in z-direction, the axis of the polymer). The wavefunctions satisfy Bloch theorem:

$$
\phi_{j}(k, r+v a)=e^{i k v a} \phi_{j}(k, r) .
$$

Here $j$ is a basis index. In addition, the energy bands and eigenstates are periodic in the reciprocal space:

$$
\begin{aligned}
E_{n}(k+l G) & =E_{n}(k), \\
\phi_{j}(k+l G, r) & =\phi_{j}(k, r) .
\end{aligned}
$$

where $G=2 \pi / a$ is the unit reciprocal vector in $z$-direction. This can be ensured if we construct wavefunctions as a linear combination of Bloch functions.

Our theoretical calculations on the electronic structure of biopolymers were based on linear combination of atomic orbitals (LCAO) (2):

$$
\Psi_{\mathrm{n}}(\mathbf{k}, \mathbf{r})=\sum_{\mathbf{j}} \mathrm{C}_{\mathrm{nj}}(\mathbf{k}) \phi_{\mathbf{j}}(\mathbf{k}, \mathbf{r}),
$$

where $\phi_{j}$ 's are the Bloch sums of the atomic orbitals $\chi_{j}$ 's:

$$
\phi_{j}(k, r)=\frac{1}{\sqrt{N}} \sum_{v} e^{i k v a} \chi_{j}\left(r-R_{i}\right) .
$$

The helical structure can be generated from its 'reduced' unit cell by applying the screw symmetry operation:

$$
S^{v}(\mathbf{r})=D(v \alpha)(\mathbf{r})+v \mathbf{a},
$$

where $D(\alpha)$ is the matrix that rotates a vector through an angle $\alpha$ about the positive $z$-axis (for a right-handed helix):

$$
D(\alpha)=\left[\begin{array}{ccc}
\cos \alpha & -\sin \alpha & 0 \\
\sin \alpha & \cos \alpha & 0 \\
0 & 0 & 1
\end{array}\right] .
$$

Now the appropriate basis functions to build up the Bloch function become:

$$
S^{v}\left(\chi_{j}\left(\mathbf{r}-\mathbf{r}_{\mathbf{i}}\right)\right)=\chi_{j}\left(S^{-v}(\mathbf{r})-\mathbf{r}_{\mathbf{i}}\right) \text {. }
$$

The quantum-mechanical structure of this homopolynucleotide system was obtained with a tight binding Hamiltonian. The energy bands and eigenstates are obtained by solving the following eigenvalues equation (we use atomic unit):

$$
(T+V) \Psi_{n}(r)=E_{n} \Psi_{n}(r),
$$

where $T=-\frac{1}{2} \nabla^{2}$ is the kinetic energy operator, 
and $V=\sum V\left(r+S^{v}\left(r_{\alpha}\right)\right)$ represents the electron-nucleus interaction potential. $v \alpha$

To evaluate the matrix elements of the potential we follow Parravicini (3):

$\left\langle\chi_{1}\left(S^{-v}(\mathbf{r})-r_{1}\right)|(T+V)| \chi_{j}\left(\mathbf{r}-\mathbf{r}_{2}\right)\right\rangle$

$=\left(E_{1}+E_{j}\right)\left\langle x_{1} \mid x_{j}\right\rangle-\left\langle x_{1}|T| x_{j}\right\rangle$

$\chi_{1}, \chi_{j}$, centered on different atoms;

$=E_{i} \delta_{i j}$

the same atom.

So the form of the potential need not be known explicitly. The atomic energy levels, the $E_{i}$ 's, were taken from very accurate calculation (4). With known energy bands, the excitation energy from an occupied state $(n, k)$ to an unoccupied one $\left(l, k^{\prime}\right)$ is:

$$
\hbar \omega(\mathbf{q})=E_{1}\left(k^{\prime}\right)-E_{n}(k) \text {. }
$$
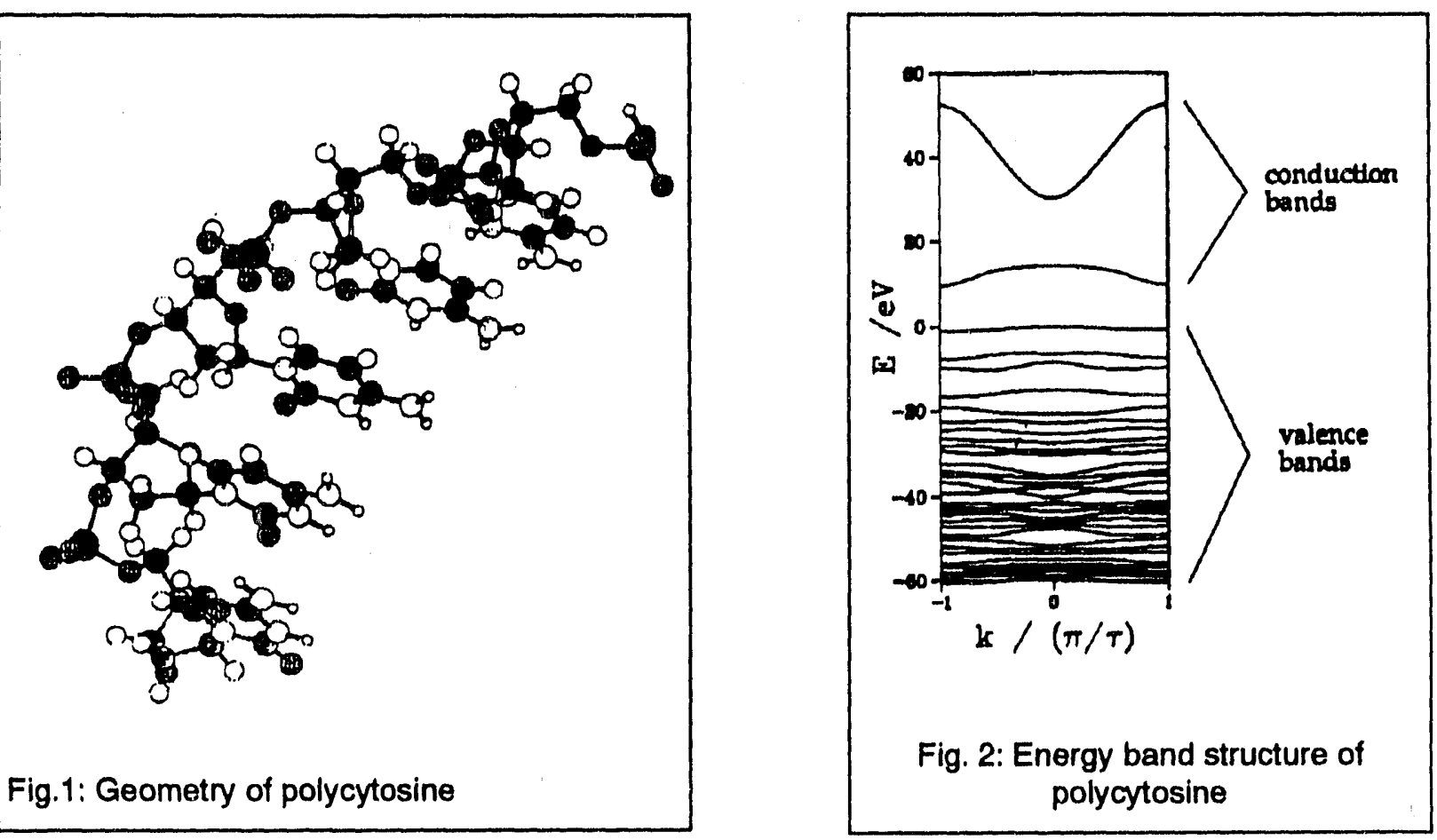

We have obtained the dielectric response function and energy-loss function for polycytosine. This is a single strand polymer with helical geometry and a cytidine monophosphate in each unit cell (Figure 1). The energy bands obtained for such a homopolynucleotide are shown in Figure 2 . The calculated dielectric response function $\left(\varepsilon=\varepsilon_{1}+i \varepsilon_{2}\right)$ for small momentum transfer $(q=0.01 / \AA$, the wave vector of a $20 \mathrm{eV}$ photon.) is shown in Figure 3, and the energy-loss function (unsmoothed and smoothed) in Figure 4, together with the measured data for DNA. The computation for polycytosine was performed on a Cray 2/8 at the National Energy Research Supercomputer Center at the Lawrence Livermore Laboratory, California. We took the poly-C geometry from cystallographic studies of B-DNA. The basis set 
used was STO-3G, and the BZ was divided into $10 \mathrm{k}$-intervals. A hydrogen atom was added to the phosphate group to neutralize the negative charge.

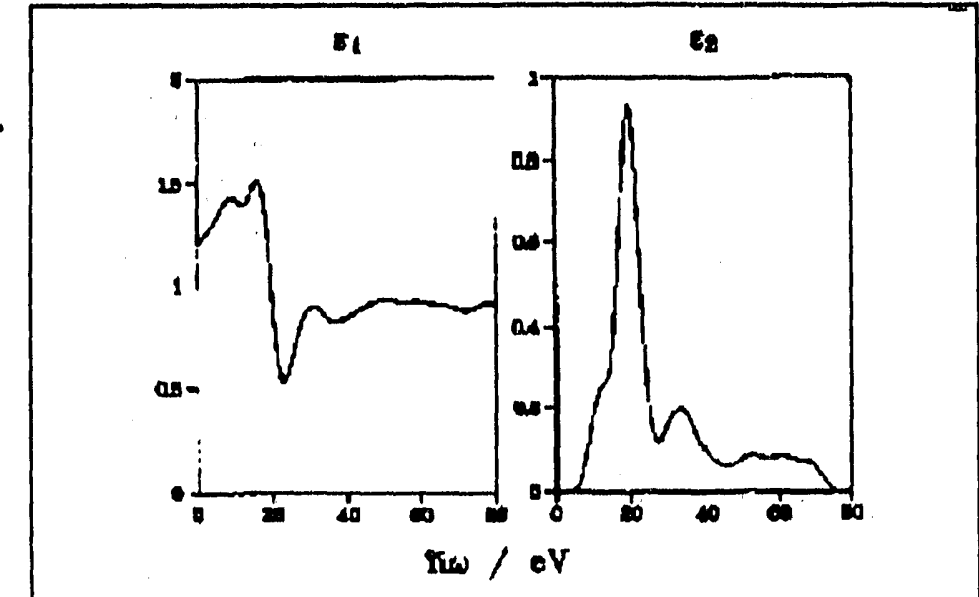

Fig. 3: Real and imaginary part of dielectric response function of polycytosine

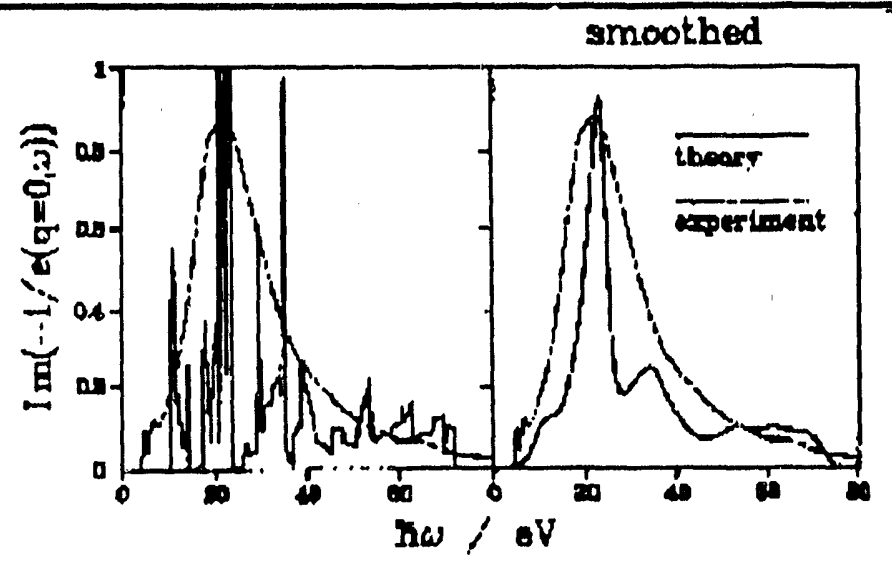

Fig. 4: Energy-loss function of polycytosine
The theoretical result consists of a lot of peaks, in contrast to the featureless experimental curve (Figure 4, left). We think that the molecular structure showed up in the theoretical calculation, but was smeared in the experiment by a complex neighboring environment. Our model assumes one helix in the whole system. In contrast, experimentalists used polycrystalline thin film, in which the DNA strands were oriented randomly with respect to the electron path. In addition, ours was a single strand, while real DNA was, of course, a double helix. So, one strand would be subject to perturbation from its complementary strand. In a word, our theoretical model was 'simpler' than the experimental geometry. Simple structures have sharp peaks, as exemplified by the spectrum of pure metals (Powell). To test our conjecture, we smoothed our calculated numbers with gaussian functions (Mintmire), and the result agreed much better with experiment (Figure 4, right). Above all, we wish to point out that the experimental thin film is no better model than the theoretical polymer for the DNA in biological tissue. DNA in the cell environment are surrounded by water, ions and other macromolecules. Thus from a radiation point of view, there is no need to strive for complete agreement with the thin film data.

Our calculation found a peak at $22 \mathrm{eV}$, remarkably close to the experimental peak seen in optical data (Figure 4, right). Similar peaks were also found in experimental data for all the nucleotide bases (5). It has been suggested quite frequently that this pronounced peak of $22 \mathrm{eV}$ represents a quanta of plasma excitation (i.e. plasmon). The question concerning the existence of plasmon is cardinal in radiation biophysics, since it relates to the localization in the polymer of the energy deposited by the incident particle. To take a (possibly) exaggerated example, a real plasmon, delocalized over some $10^{6}$ nucleotides, will all but eliminate the effect of the spatial 
distribution of the energy-transfer events. (Should this be correct one would have $\mathrm{RBE}=1$ for all radiations).

The fact that this same peak is found on just the base molecule (6) seems to rule out the existence of plasmon, since plasmon cannot exist in a molecule. The energy of the peak, $22 \mathrm{eV}$, corresponds to a free-electron gas plasmon, where the electron density (the only parameter involved) corresponds to the DNA strand. This is, of course, unrealistic. An improved, yet still approximate calculation indicates plasmon energies of $6 \mathrm{eV}(7)$ or $16 \mathrm{eV}$ (8). These are both in conflict with the interpretation of the experimental spectrum of plasmon excitation. The mathematical definition of the plasmon is $\varepsilon_{1}(q, \omega)=\varepsilon_{2}(q, \omega)=0$. One of these conditions $\left(\varepsilon_{2}=0\right)$ may be relaxed, if the plasmon has a finite lifetime. Figure 3 indicates that these conaitions are not satisfied. Ehrenreich and Philip (9) have presented somewhat modified conditions for defining a plasmon, yet their conditions are not satisfied either. At this stage, in our investigation we must conclude that there is little support for the notion of an electronic plasmon in DNA. It may be that the whole notion of 'plasmon' is ill-defined when --- as is the case here ---its lifetime is $10^{-16} \mathrm{sec}(10)$ (note that this is 100 times shorter than a $\pi^{0}$ meson); whether or not one is to call this excitation 'particle' becomes mere semantics.

\section{REFERENCES}

1. H. Ehrenreich and M.H. Cohen, Self-consistent field approach to the manyelectron problem. Phys. Rev. 115, 786-790 (1959).

2. J. Callaway, Quantum Theory of the Solid State, 2nd ed. Academic Press, San Diego, 1991.

3. G. P. Parravicini and L. Resca, Electronic states and optical properties in cubic ice, Phys. Rev. B8, 3009-3023 (1973).

4. W.A. Harrison, Electronic Structure and the Properties of Solids, Dover, New York, 1989.

5. M.W. Williams and E.T. Arakawa, Optical and dielectric properties of materials relevant to biological research. In Handbook on Synchrotron Radiation (E. Rubenstein, S. Ebashi and M. Koch Eds.) Vol. 4, Elsevier, New York, 1990.

6. M. Dillon, private communication, 1992.

7. J. Jager and J. Ladik, Estimation of the excitation energy of the low-lying collective excited state for different periodic DNA models, Phys. Lett. 28A, 328329 (1968).

8. J. Ladik, private communication, 1992. 
9. H. Ehrenreich and H. R. Philip, Optical properties of semiconductors in the ultra-violet. In Proc. Int. Conf. Phys. Semiconductors (A. C. Strickland Ed.) Bartholomew Press, U.K.

10. J. Bednar, Electronic excitation in condensed biological matter, Int. J. Radiat. Biol. 48, 147-166 (1985). 


\section{BIOPHYSICAL MODELLING}




\title{
A NON-PARAMETRIC, MICROdOSIMETRIC-BASED APPROACH TO THE EVALUATION OF THE BIOLOGICAL EFFECTS OF LOW DOSES OF IONIZING RADIATION
}

\author{
Marco Zaider and M. Varma
}

\section{Introduction}

A central theme in radiation protection is the establishment of a link between the physical characteristics of a radiation field and the probability of exposure-related deleterious effects on humans. The procedure currently in effect consists of defining at each dose $D$ of a certain radiation a quantity termed close equivalent $H$, which is numerically equal to the absorbed dose of low-LET radiation producing equal biological effect. It is postulated that $H$ and $D$ are linearly related, the proportionality constant, $Q$, being a function of the "quality" of the radiation only:

$$
\mathbf{H}=\mathbf{N} \cdot \mathbf{Q} \cdot \mathbf{D} \text {. }
$$

$\mathrm{N}$ is a factor meant to account for effects other than radiation quality; it is currently taken as 1. The meaning of "quality" in the context of $\mathrm{Eq}(1)$ is certainly the crucial aspect in the definition of $\mathrm{H}$. Before addressing this, however, several things should be noted. A linear relationship between $\mathrm{H}$ and $\mathrm{D}$ implies that dose-effect curves for all radiations involved are also linear. This is generally not the case unless the absorbed dose, D, is restricted to the range of so-called "low doses". For a given radiation an unambiguous definition of this range can be given only in terms of its microdosimetric spectrum in a volume which simulates the sensitive target: a dose is "low" when the probability of more than one event (i.e. particle traversal with concomitant energy deposition) in the sensitive target is negligible. The condition is:

$$
\mathrm{n}=\mathrm{D} / \mathrm{z}_{1 \mathrm{~F}}<<1,
$$

where $z_{1 F}$ is the average specific energy per event (see below), and therefore $n$ is the mean number of events at dose D. The term "low dose" is itself dependent on the quality of the radiation. For instance, taking - quite arbitrarily - $\mathrm{Eq}(2)$ to mean $\mathrm{n} \leq 0.1$, a tissue-equivalent spherical volume of $1 \mu \mathrm{m}$ diameter exposed to $1 \mathrm{~Gy}$ of $1-\mathrm{MeV}$ neutrons qualifies for low-dose exposure, but not when irradiated with $1 \mathrm{~Gy}$ of $x$ rays.

What makes $Q$ different from $R B E$, to which it is conceptually related, is the fact that its numerical values are weighted averages of RBE's for those effects deemed important in radiation protection. Since no scientific method exists as yet for setting the weighting factors )they are assigned on the basis of personal judgment in appropriate forums) $Q$ itself does not represent - at least in our view - a scientific quantity and it will not be further considered here. $q$ for specific end points (we use the lower case letter to emphasize this distinction) is generally obtainable by performing measurements and assessing the low-dose limit of the slope of the dose- 
effect curve The problem is reduced then to finding a procedure by which q could be extrapolated to fields for which radiobiological data do not exist.

Currently $Q$ is defined in terms of the LET of the radiation. The shortcomings of this particular choice have been extensively discussed (ICRU-40, 1986). These deficiencies have led two groups of investigators (Bond and Varma, 1982; Zaider and Brenner, 1985) to suggest, independently, the quantity lineal energy, $y$, (or its equivalent, specific energy, $z$ ) as a descriptor of the field. Specifically, it has been proposed that, for a given end point, the specific quality factor $\bar{q}$ of a radiation be calculated from the dose distribution in lineal energy according to:

$$
\bar{q}=\int q(y)\left[y f_{1}(y) d y / y_{F}\right]
$$

where $q(y)$ is an end-point-dependent function, $f_{1}(y) d y$ is the single-event microdosimetric distribution in the reference volume, and $y_{F}$ is the frequencyaveraged lineal energy:

$$
y_{F}=\int y f_{1}(y) d y / \int f_{1}(y) d y
$$

$\mathrm{q}(\mathrm{y})$ has been termed specific quality function (SQF) by Zaider and Brenner (1985). In the formalism suggested by Bond and Varma (1982) the quantity $q(y) \cdot y$ is used instead; this is termed hit-size effectiveness function.

Given $\mathrm{q}(\mathrm{y})$, the determination of $\overline{\mathrm{q}}$ amounts to measuring the microdosimetric spectrum, $f_{1}(y)$, of the radiation field. $q(y)$ is an empirical function which gives the biological effectiveness at lineal energy $y$. It can be generally obtained by exposing a system to radiations, $i$, characterized by microdosimetric spectra $\left.f_{i}(y), b\right)$ determining the initial slope, $\alpha_{i}$, for each resulting dose-response curve, and c) by solving the system of equations:

$$
\alpha_{i}=\int q(y)\left[\frac{y f_{i}(y) d y}{y_{F} i}\right] \quad i=1,2, \ldots
$$

The requirement that $q(y)$ be non-negative and the fact that $\alpha_{i}$ are affected by experimental uncertainties makes it highly unlikely that a solution to $\mathrm{Eq}(5)$ can be found. The expression, $\mathrm{Eq}(5)$, is clearly an approximation to the exact doseresponse dependency of the system.

Approximate solutions, $q(y)$, may be obtained by either postulating an analytic expression for this quantity, or by discretizing $q(y)$ in a set $\left\{q_{1}=q\left(y_{1}\right), q_{2}=q\left(y_{2}\right), \ldots\right\}$. Agreement between the left and right-hand side terms of $\mathrm{Eq}(5)$ is then sought only in the least-squares sense, that is by minimizing the functional: 


$$
F[q(y)]=\sum_{i}\left|\alpha_{i}-\int q(y)\left[\frac{y f_{i}(y) d y}{y_{F}^{1}}\right]\right|^{2} .
$$

Both methods have been tried for severald end points (Varma and Bond, 1982; Zaider and Brenner, 1985; Zaider and Brenner, 1986) with somewhat ambiguous results. In the case of a parametric formulation of $q(y)$ the solution is totally dominated by the analytic form selected. Non-parametric solutions, on the other hand, have highly irregular shapes and smoothing techniques need to be utilized. In either case there appear to be no consistent method for estimating the goodness of the fit of $\mathrm{Eq}(6)$ on the space of possible sets $\left\{\mathrm{q}_{\mathrm{i}}\right\}$.

In this paper we describe a novel, bias-free method for the determination of $q(y)$. This method is based on the application of maximum entropy and Bayesian approaches to solve the inverse problem, $\mathrm{Eq}(5)$.

The determination of $a(z)$

We attempt to solve the following discretized version of $\mathrm{Eq}(5)$ :

$$
\alpha_{i}=\sum_{j=1}^{N} q_{j} \cdot d_{j i}, \quad i=1,2, \ldots, M
$$

where an obvious notation has been introduced for $d_{j i}$. In general $M<<N$ and the system of equations (7) is underdetermined. To select an optimal solution (optimal means: consistent with all constraints on $q_{j}$, such as positivity, and unbiased) we use Bayes' theorem:

$$
\mathrm{P}\left(\mathrm{q}_{\mathrm{j}} \mid \alpha_{\mathrm{i}}, \mathrm{I}\right)=\mathrm{P}\left(\mathrm{q}_{\mathrm{j}} \mid \mathrm{I}\right) \cdot \mathrm{P}\left(\alpha_{\mathrm{i}} \mid \mathrm{q}_{\mathrm{j}}, \mathrm{I}\right) / \mathrm{P}\left(\alpha_{\mathrm{i}} \mid \mathrm{I}\right) .
$$

In this expression $P(x \mid y, z, \ldots)$ means the conditional probability of $x$ given $y, z, \ldots$ The theorem, $\mathrm{Eq}(8)$, states that the probability of a certain solution $\left\{q_{j}\right\}$ given the data $\left\{\alpha_{i}\right\}$ and any other information I (restrictions) on $q_{j}$, is proportional to the prior probability of $\left\{q_{j}\right\}$ and the likelihood of $\alpha_{i}$, should $q_{j}$ be indeed the correct choice.

If $\alpha_{i}$ are known exactly then $P\left(\alpha_{i} \mid q_{j}, I\right)=1$ if $\left\{q_{j}\right\}$ satisfy Eq (7), and zero otherwise. Since $\alpha_{\mathrm{i}}$ are in fact subject to experimental uncertainties the restriction imposed by $\mathrm{Eq}(7)$ should be written as:

$$
\alpha_{i}=\sum_{j=1}^{N} q_{j} d_{j i}+r_{i}, \quad i=1,2, \ldots, M
$$


where a "noise" term, $r_{i}$, has been introduced. It is customary to take $r_{i}$ as being normally distributed according to $\mathrm{N}\left(0, \sigma_{\mathrm{i}}\right)$. Under these conditions the likelihood function in $\mathrm{Eq}(8)$ becomes:

$$
P\left(\alpha_{i} \mid q_{j}, I\right)=\exp \left[-\frac{1}{2} \sum_{i=1}^{M}\left(\alpha_{i}-\sum_{j=1}^{N} q_{j} d_{j i}\right)^{2} / \sigma_{1}^{2}\right]=\exp \left[-\chi^{2}\right] .
$$

As regards the prior probability, $P\left(q_{j} \mid \alpha_{i} I I\right)$, the common approach is to take this quantity equal to 1 (as long as $q_{j}$ are non-negative) and therefore make the assumption that with no prior information all sets $\left(q_{j}\right)$ are equally probable. This is incorrect. Indeed, let

$$
Q_{0}=\sum_{j=1}^{N} q_{j}
$$

The quantities $\mathrm{q}_{\mathrm{j}} / \mathrm{Qo}$ may be interpreted as representing a probability distribution. According to Shannon (1948) and Jaynes (1982) the amount of uncertainty in this (or any other probability distribution is given by its entropy, $\mathrm{H}$ :

$$
H=- \text { const } \cdot \sum_{j=1}^{N}\left(\frac{q_{i}}{Q_{0}}\right) \ln \left(\frac{q_{i}}{Q_{0}}\right) \text {. }
$$

It is clear now that the least-biased (i.e. most non-committal) prior choice of $\left\{q_{j}\right\}$ would be that which maximizes $\mathrm{H}$, that is allows for maximum uncertainty. For, any other set (of correspondingly smaller $\mathrm{H}$ ) would presume that we have some additional data on $\mathrm{q}$. This procedure is known as the maximum entropy approach.

The application of Bayes' theorem amounts now to finding the set $\left\{q_{j}\right\}$ which maximizes:

$$
\mathrm{L}\left[\mathrm{q}_{\mathrm{j}}\right]=\mathrm{H}-\lambda \cdot \chi^{2},
$$

where $\lambda$ is a constant and $\chi^{2}$ is defined in $\mathrm{Eq}(10)$. This can be achieved with the aid of the Lagrange multiplier technique. The maximum entropy solution may be supplemented with smoothing conditions (Zaider and Minerbo, 1988).

\section{Results}

We report here specific quality factors, $q(z)$, obtained by analyzing data on cellular inactivation, chromosome aberrations, mutation and cellular transformation. The experimental results have been selected on the basis of a) relevancy for radiation protection, and $b$ ) the number of different radiations used. A common problem in performing these analyses is the unavailability of microdosimetric spectra, 
particularly for high-LET radiation. Unless measured spectra were available we have used the track segment approximation in estimating the microdosimetric distributions. This has a certain degree of justification given that: a) the radiations consisted of mono-LET charged particles, and $b$ ) the site diameter selected, $1 \mu \mathrm{m}$, is fairly large compared to the radial extension of the tracks. Nevertheless, because of this approximation the results reported here should be considered tentative only.

The following data sets have been analyzed: (1) Inactivation of synchronized (late S) Chinese hamster V79 cells (Bird et al, 1980); charged particles in the range 10$170 \mathrm{keV} / \mu \mathrm{m}$ have been used, (2) Chromosome aberrations in human lymphocytes; these data have been analyzed previously (Zaider and Brenner, 1985) using a nonparametric technique, (3) Oncogenic transformation of C3H/10T1/2 cells (Heil et al, 1988); charged particles in the range 10 to $120 \mathrm{keV} / \mu \mathrm{m}$, and $x$ rays have been used, (4) Mutation and inactivation of human diploid fibroblasts. $x$ rays, $\gamma$ rays and 20$470 \mathrm{keV} / \mu \mathrm{m}$ charged particles have been used; an analysis of the mutation data has been reported using the same non-parametric method mentioned above (Cox et al, 1977; Zaider and Brenner, 1986), (5) Chromatid exchanges in $\mathrm{CH}_{2} \mathrm{~B}_{2}$ cells (Skarsgard et al, 1967); the LET of the charged particles ranged from 1.25 to $351 \mathrm{keV} / \mu \mathrm{m},(6)$ Neoplastic cellular transformation and inactivation of C3H10T1/2 cells (Yang et al, 1985); data are in the range of 2.6 to $1900 \mathrm{keV} / \mu \mathrm{m}$.

Results are shown in Fig. 1 in a $q(y)$ versus y representation. $q(y)$ is normalized so that for low-LET radiation $R B E=1$. When all the curves, other than that corresponding to human lymphocytes, are polled quite similar shapes (and even semi-quantitative agreement) is noticeable, indicating the extent to which the spatial pattern of energy deposition (as measured by y) rather than the specific cellular system or end point determine the quality factors. This relative independency of $\mathrm{q}(\mathrm{y})$ of the end point is prominent when, for the same cell line (e.g. human fibroblasts), two different effects (mutation and cellular inactivation) are compared. The curve $q(y)$ for human lymphocytes appears to be different from all the rest. We have no explanation for this results.

\section{Discussion and conclusion}

The proposal that quality factors be calculated from a microdosimetric function $\mathrm{q}(\mathrm{z})$ must rest, among other things, on the assumption that the amount of energy deposited in the sensitive target determines the effect. This assumption, known as the site model, although widely used in biophysical modeling, has been questioned following experiments with correlated ion beams (Rossi, 1979). It has been proposed that a "distance" model (i.e. where the relative separation of energy transfers, rather than their total number, determines the biological outcome) may describe more accurately the effects of ionizing radiation. The formal expression of this statement is the generalized theory of dual radiation action (Kellerer and Rossi, 1978). Here the initial slope is expressed as: 


$$
\alpha \sim \int \frac{P_{1}(x) \cdot P_{2}(x)}{4 \pi x^{2}} \cdot g(x) d x
$$

where $P_{1,2}(x)$ represent proximity functions for the track rand sensitive target, respectively, and $g(x)$ is the probability that sublesions a distance $x$ apart interact. It has been noted that, since only the product $P_{2}(x) \cdot g(x)$ is amenable to experimental determination, one may very well define a sensitive target for which $P_{2}{ }^{\prime}(x)=P_{2}(x) g(x)$ and thus return (with no experimentally detectable differences) to the site model. Put it differently, to the extent that the shape of the sensitive target can be chosen freely one should be able to select a geometry whose microdosimetric spectrum determines indeed the initial slope. As a matter of principle then the site model remains valid.

It is clear, however, that in terms of experimental microdosimetry very few geometries (sphere, cylinder) are acceptable and therefore practical considerations restrict significantly the choice of an equivalent sensitive site. It has been proposed (Zaider and Rossi, 1988), and this may be very relevant for $q(y)$, that a series of microdosimetric spectra in spheres of different diameters may be used instead. Whether such more sophisticated tools are indeed useful in radiation protection remains to be determined.

The application of the same technique (Bayes and maximum entropy) to a large number of experimental data sets - to our knowledge performed here for the first time - reveals a surprising uniformity in the functions $q(y)$ and therefore supports the concept of a microdosimetric-based quality factor. It also point out to the acute need for experimental microdosimetry for all radiations of interest in radiation protection.

\section{References}

Bird PR, Rohrig N, Colvett RD, Geard CR, Marino SA 1980 Radiat Res 82277.

Bond VP and Varma MN 1982 CEC Report EUR-8395 p423.

Cox R, Thacker J, Goodhead DT and Munson R 1977 Nature 267425.

Hei TK, Komatsu K, Hall EJ and Zaider M. 1988 Carcinogenesis 9747.

ICRU-40 1986 The Quality Factor in Radiation Protection (ICRU, Bethesda Ma).

Jaynes ET 1982 Proc. IEEE 70939.

Kellerer AM and Rossi HH 1978 Radiat Res 75471.

Rossi HH 1979 Radiat Res 78185.

Shannon CE 1948 Bell Systems Tech J 27379.

Skarsgard LD, Kihlman BA, Parker L, Pujara CM and Richardson S 1967 Radiat Res Sup Z 208. 
Varma MN andBond VP 1982 CEC-Report EUR-8395 p439.

Yang TC, Craise LM, Mei M and Tobias CA 1985 Radiat Res 104 S177.

Zaider M and Brenner DJ 1985 Radiat Res 103302.

Zaider M and Brenner DJ 1986 Radiat Prot Dos 1579.

Zaider M and Minerbo GN 1988 Phys Med Biol 331273.

Zaider M and Rossi HIH 1988 Radiat Res 11315.

Fig. 1: $q(y)$ as a function of $y$ for eight data sets (end Points) as indicated. The parametric analysis of Varma and Bond (1982) is also shown. 

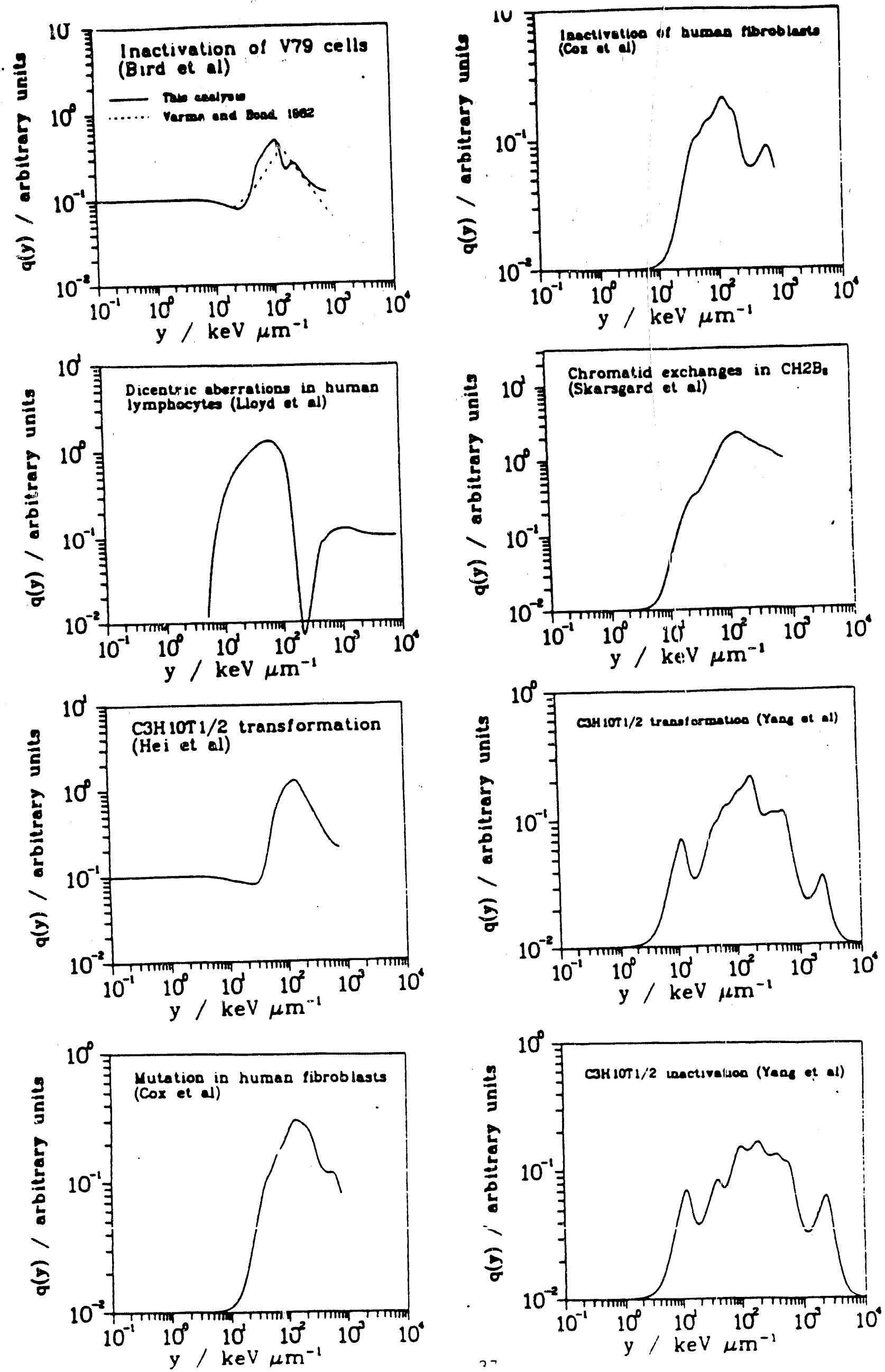


\section{High-Let Radiation Risk Assessment at Medium Doses}

\section{J. Brenner}

Environmentally, most of the radiation exposures to which humans are exposed are in the low-dose range, defined as occurring when the average number of radiation tracks traversing the cell nucleus is much less than one. In this situation, an increase in dose simply results in a proportionate increase in the number of cells undergoing a single energy-deposition event, and thus the effect should increase linearly with dose.

Thus there is a coherent rationale for the present system of low-dose radiation protection in which the risk is related to the dose equivalent, $H$, defined as

$$
\mathrm{H}=\mathrm{D} \times \mathrm{Q} \text {. }
$$

Here $D$ is the dose and $Q$ is a dimensionless quantity, sometimes called the radiation weighting factor $w_{r}$, reflecting the biological effectiveness (for "relevant" endpoints) of single tracks of different radiations.

At higher doses, but still in the dose region where carcinogenesis is the dominant concern, this rationale breaks down; even a cursory glance at experimental results for radiation-induced carcinogenesis in vivo strongly suggests that the slope of dose-response relations decreases with increasing dose. This same conclusion can be drawn from measured dose-dependent probabilities for the induction of oncogenic transformation per exposed cell in vitro.

Because most radiation exposures are in the low-dose range, such effects are of limited importance, although they do provide the potential for erroneous extrapolations to low doses. There are, however, situations where higher doses are delivered, such as a very high domestic radon exposure, an accidental radiation exposure, or a manned mission to Mars. A 3-year Mars mission has been estimated to result in a dose to the astronauts of at least $0.5 \mathrm{~Gy}$ of radiations of widely-varying LET.

For densely-ionizing radiations, the evidence is strong that at doses below $1 \mathrm{~Gy}$ and, in fact, usually below $0.5 \mathrm{~Gy}$, the dose-response relation (prevalence in-vivo, or oncogenic transformation per exposed cell in vitro) will reach a maximum value. In vivo, at higher doses, in some cases the adjusted incidence remains approximately at a plateau value, whilst in other cases the incidence decreases as the dose is further increased.

It is clear that dose-response relations for prevalence in vitro and transformation per exposed cell in vitro generally exhibit a maximum value as the dose increases. A plausible model for such dose-response relations was suggested in 1965 by Gray. He suggested that the overall incidence was the result of two processes, transformation induction and cellular inactivation. Thus the incidence per exposed cell can be written 


$$
I(D)=T(D) \times S(D)
$$

where $T(D)$ is the transformation inidence per surviving cell, and $S(D)$ is the proportion of surviving cells.

If both $T$ and $S$ can be described by the linear-quadratic formalism, then

$$
I(D)=\left(\alpha_{T} D+\beta_{T} D^{2}\right) \exp \left(-\alpha_{S} D-\beta_{S} D^{2}\right)
$$

where $\left[\alpha_{T}, \beta_{T}\right]$ and $\left[\alpha_{S}, \beta_{S}\right]$ are constants referring, respectively, to transformation induction and cellular survival. This model was described - perhaps with some degree of overstatement - in NCRP report 64 as "the general dose-response relationship currently favored by radiation biologists for radiation-induced cancer".

There is a strong mechanistic rationale for the linear-quadratic relations described by Eq. (3); both cellular survival, through asymmetric exchange-type chromosomal aberrations, and oncogenesis, through symmetric chromosomal exchanges or interstitial deletions, are two-hit type phenomena. Specifically, for cell killing there is strong evidence that the dominant mechanism involves asymmetric exchange-type chromosome-aberration formation, for example dicentric induction. In terms of oncogenesis the evidence for most hematological cancers also implicates exchange-type chromosomal aberrations (translocations, probably associated with oncogene activation; solid tumors are more often associated with deletion-type mutational damage (removal of "suppressor" genes). Exchange-type aberrations (dicentrics and translocations) and interstitial deletions are both two-hit phenomena. Thus a linear-quadratic formalism, though with different parameters for each, is likely to apply both to oncogenesis and cell killing.

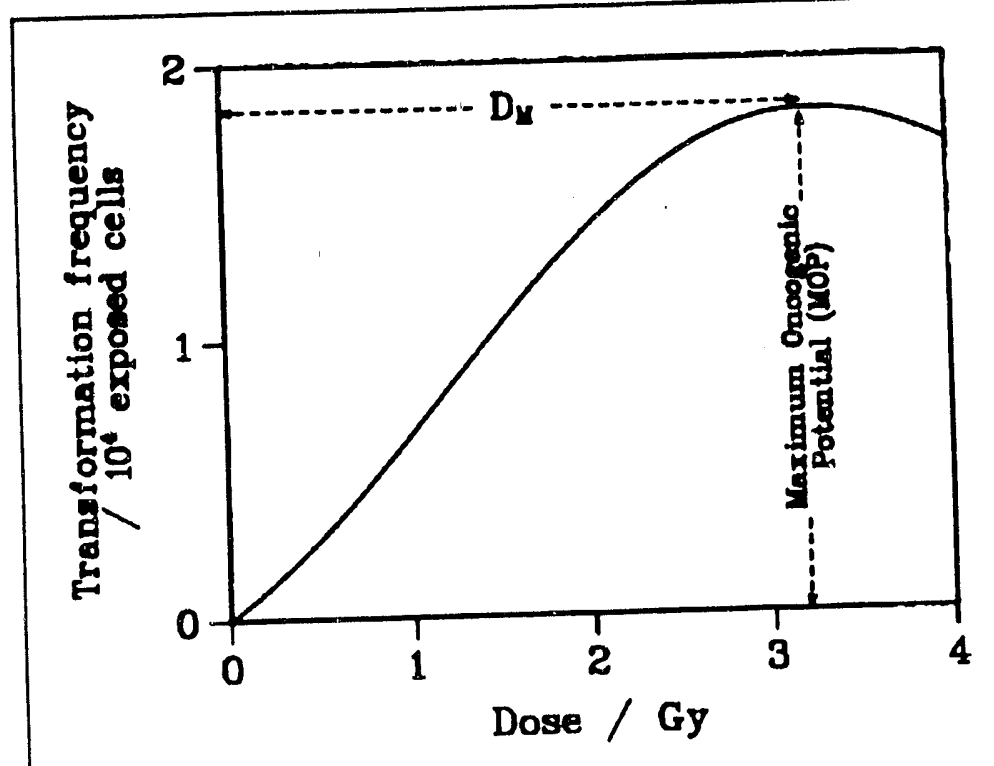

Fig. 1: Shape of dose-response relation according to Eq. (3).

interplay between the parameters $\left[\alpha_{S}, \beta_{S}\right]$ and $\left[\alpha_{T}, \beta_{T}\right]$.
The shape of the dose-response relationship described by Eq. (3) has been extensively discussed. As illustrated in Fig. 1, there is an initial linear rise in incidence followed by a super-linear rise, followed by an decrease in slope and, ultimately, a decrease in incidence. The specific features of the dose-response, for example the visibility of the superlinear increase, the position of the peak incidence, and the subsequent rate of decrease of the incidence, depend on the 
Absolute values of incidence are end-point specific, depending also on such phenomena as hormonal, immunological and tissue effects. The dose-dependence of these effects is not known, but it is a testable hypothesis that it can be incorporated into the linear-quadratic incidence term in Eq. (3), with appropriate parameters $\left[\alpha_{\mathrm{T}}, \beta_{\mathrm{T}}\right]$. Such an assumption is testable with reference to experimental data in vivo.

It is the purpose of this note to point out some testable simplifications of Eq. (3) which may be of practical use in risk assessment at doses of high-LET radiation above $\sim 0.5 \mathrm{~Gy}$, where it cannot be assumed that risk increases linearly with dose.

If we assume that the incidence is given by Eq. (3), then the maximum possible incidence, which we trim the maximum oncogenic potential (MOP), will occur at a dose, $D_{M}$, where the derivative of the incidence with respect to dose, is zero (see Fig. 1). Thus, diff $九$ rentiating Eq. (3) we obtain

$$
\frac{1}{\alpha_{\mathrm{S}}}(\alpha / \beta)_{\mathrm{T}}+D_{\mathrm{M}}\left[\frac{2}{\alpha_{\mathrm{S}}}-(\alpha / \beta)_{\mathrm{T}}\right]-D_{\mathrm{M}}^{2}\left[1+2(\alpha / \beta)_{\mathrm{T}} /(\alpha / \beta)_{\mathrm{S}}\right]-2 \mathrm{D}_{\mathrm{M}}^{3} /(\alpha / \beta)_{S}=0 .
$$

This cubic equation has no simple analytic solution, but can be solved numerically for $D_{M}$, the dose at which the maximum oncogenic potential (MOP) occurs, and therefore for the MOP itself.

It should be emphasized that we are not now considering the risk itself, but the maximum possible value of the risk (see Fig 1). We now investigate how this maximum oncogenic potential (MOP) might be expected to vary with LET. We shall make two assumptions, both of which are consistent with experiment:

First, we assume that, for a given biological system, the quadratic parameters $\beta_{S}$ and $\beta_{\mathrm{T}}$ in Eq. (3) are independent of LET, leaving the LET dependence in the linear $(\alpha)$ terms. There is good evidence, both theoretical and experimental, for this assumption. Mechanistically, it follows directly from the interpretation of the linear-quadratic formalism, in which the linear term describes damage from a single track of radiation, whilst the quadratic term describes damage caused by the effects of two independent tracks. Given that the linear term describes single-track action, it will be dependent on the energy-deposition characteristics of that track, i.e. the radiation quality or LET. On the other hand, the quadratic term describes multi-track action from independent energy depositions in different tracks, and so cannot depend on the track structure or LET.

The experimental evidence for the assumption that the quadratic terms are independent of radiation quality is strong for survival and chromosomal-aberration endpoints in vitro, where accurate experiments can be performed to test this hypothesis. Currently, oncogenic-transformation experiments either in vitro and in vivo are not accurate enough to test critically the hypothesis, but available data are consistent with this notion; it should be noted, however, that if the commonly-held mechanistic notions (see above) that radiation oncogenesis derives from 
chromosomal translocations or interstitial deletions are correct, we have strong reasons to expect that the quadratic terms would not vary with LET.

Our second assumption here is that, as the LET increases, the linear parameters for survival and transformation, $\alpha_{S}$ and $\alpha_{T}$, increase proportionately to each other, i.e.

$$
\alpha_{\mathrm{T}} / \alpha_{\mathrm{S}}=\mathrm{k}
$$

where $k$ is a constant. $\alpha_{S}$ is proportional to the low-dose RBE for survival, and $\alpha_{T}$ is proportional to the low-dose RBE for oncogenic transformation (per surviving cell). Eq. (5) is equivalent to the suggestion that the low-dose RBE varies in the same way with LET for survival as for oncogenic transformation. Where systematic simultaneous experiments to measure RBE have been performed for cellular survival and oncogenic transformation, this suggestion has been confirmed.

Overall, there is good evidence for our two assumptions, both of which are Overall, there is good evidence for our the assumptions, some simple mathematical
eminently testable. Given these
manipulation of Eq. (4) gives the result that, for large values of $\alpha_{S}$ - we discuss how large below - the maximum possible value of the oncogenesis rate (the maximum oncogenic potential, MOP) is independent of $(\alpha / \beta)_{S}$ and, since $\beta_{T}, \beta_{S}$ and $\alpha_{T} / \alpha_{S}$ are constant, independent of $(\alpha / \beta)_{\mathrm{T}}$. In fact the maximum oncogenic potential is

$$
\mathrm{MOP}=\left(\alpha_{\mathrm{T}} / \alpha_{\mathrm{S}}\right) / \mathrm{e}+\mathbf{O}\left(1 / \alpha_{\mathrm{S}}^{3}\right),
$$

where the notation $\mathbf{O}\left(1 / \alpha_{\mathrm{S}}^{3}\right.$ ) ("order of" $\left.1 / \alpha_{\mathrm{S}}^{3}\right)$ means terms that contain factors of $1 / \alpha_{S}^{3}, 1 / \alpha_{S}^{4}$, etc; thus for large values of $\alpha_{S}$,

$$
\text { MOP } \approx\left(\alpha_{\mathrm{T}} / \alpha_{\mathrm{S}}\right) / \mathrm{e}
$$

i.e. the constant value $k / e$. Similarly, the dose, $D_{M}$, at which this maximum incidence will occur is

$$
D_{M}=1 / \alpha_{S}+\mathbf{O}\left(1 / \alpha_{S}^{2}\right)
$$

and for large values of $\alpha_{S}$,

$$
D_{M} \approx 1 / \alpha_{S}
$$

The range of validity of the approximation of Eq. (7) is illustrated in Figs. 2, where numerically-exact calculations of the MOP are compared with this approximation. Figs. $2(a-c)$ refer to a range of reasonable values of the constant $(\alpha / \beta)_{S} /(\alpha / \beta)_{T}$. The calculations are for values $\beta_{S}$ and $\beta_{\mathrm{T}}$ of, respectively, 0.06 and $0.3 \mathrm{~Gy}^{-2}$. These are reasonable values (see legend), but comparably-shaped curves are obtained for other values of the $\beta$ parameters. 


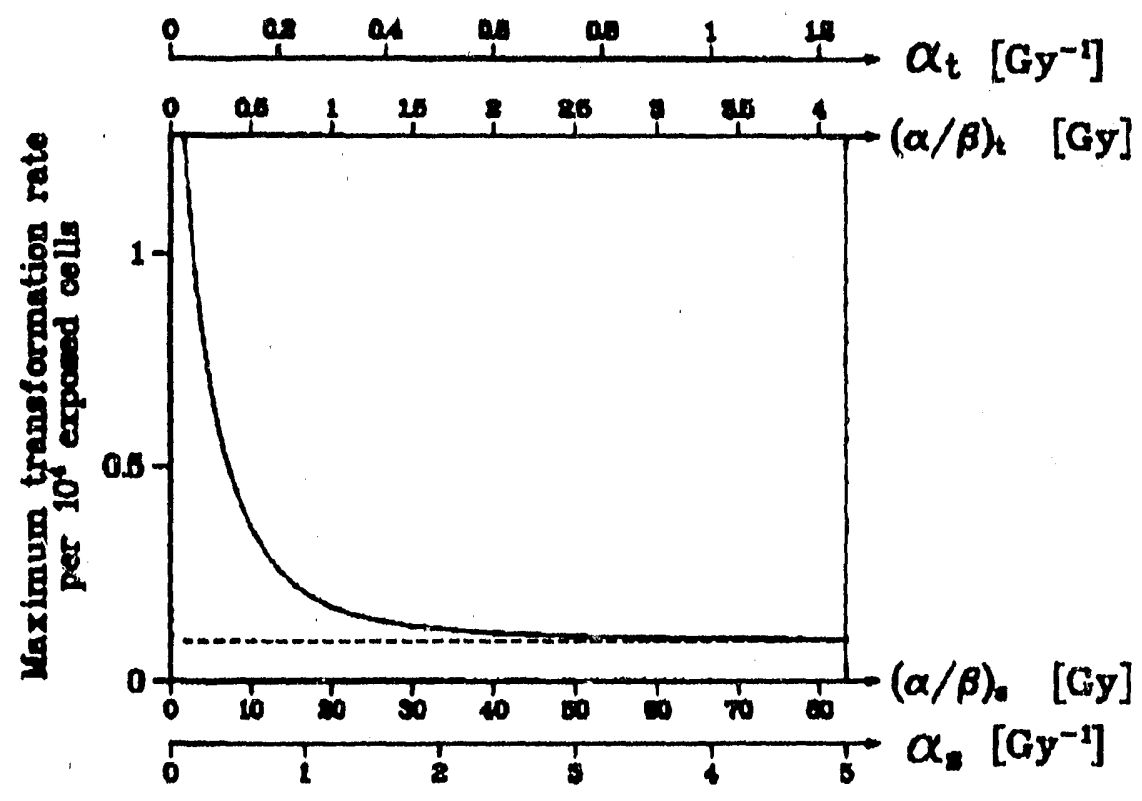

Fig. 2(a)

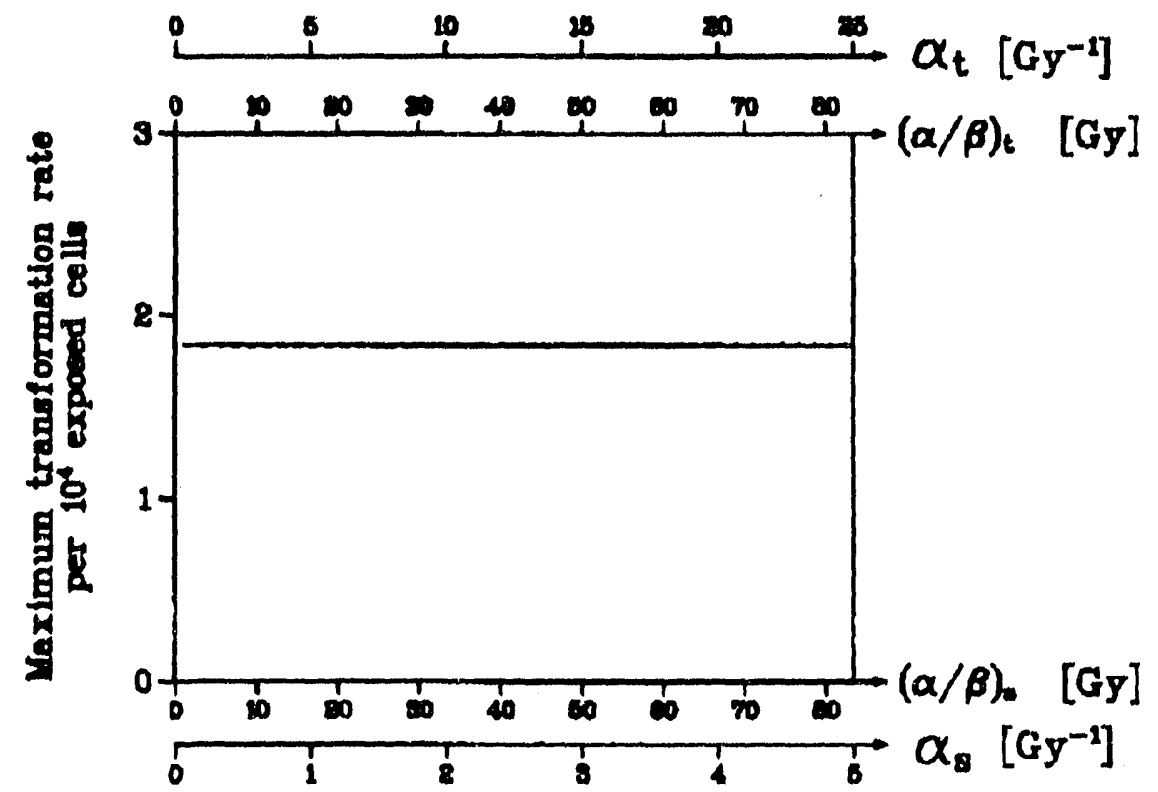

Fig. 2(b) 


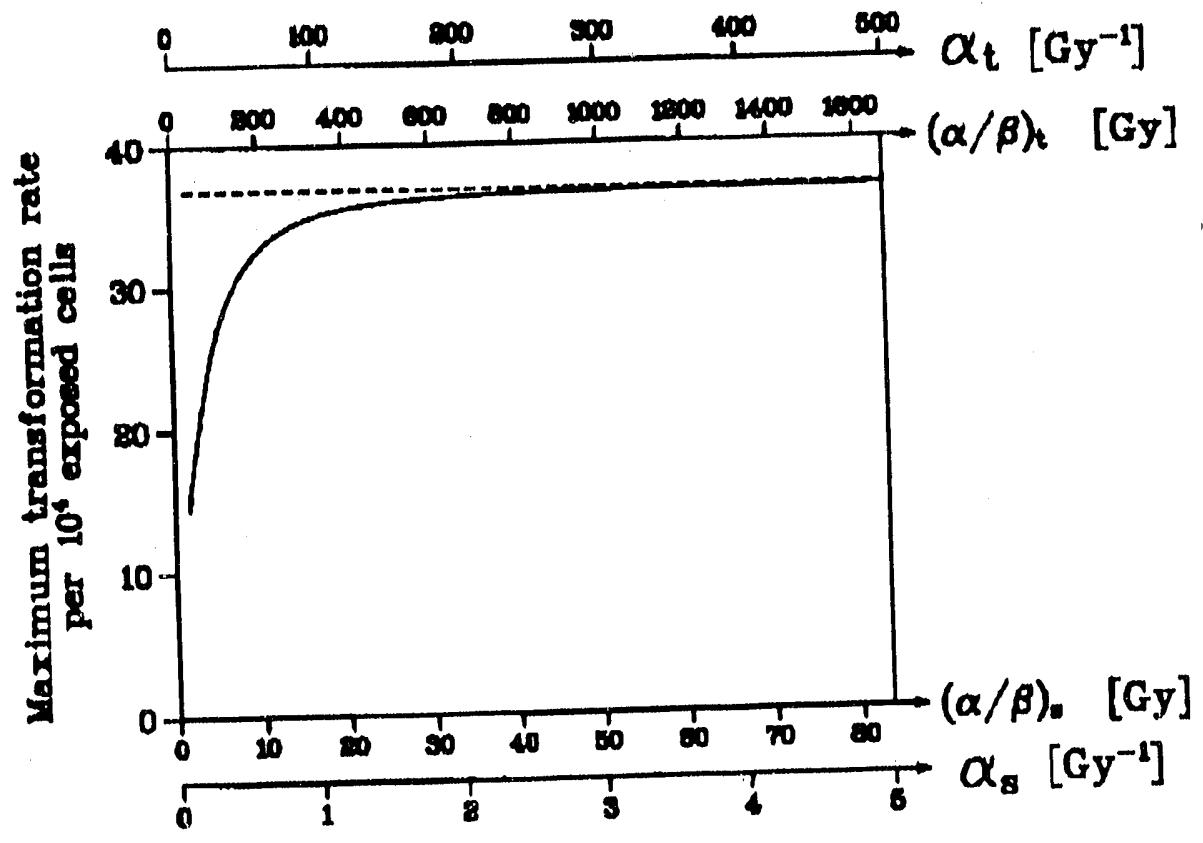

Fig. 2(c)

Fig 2: Maximum oncogenic transformation, te (MOP, full curve) as a function of increasing low-dose RBE. The dashed lines show the approximation of Eq. (7). $\beta$ values used are .06 and $.3 \mathrm{~Gy}^{-2}$ for survival and transformation respectively. Increasing $(\alpha / \beta)_{S},(\alpha / \beta)_{T}, \alpha_{S}$ or $\alpha_{T}$ correspond to increasing RBE, and thus to increasing LET. A typical value of $(\alpha / \beta)_{S}$ for low-LET $\gamma$ rays is 10 Gy. The three figures are for a representative range of values of $(\alpha / \beta)_{T}$ relative to $(\alpha / \beta)_{S}$ :

$(2 a):(\alpha / \beta)_{S} /(\alpha / \beta)_{T}=20$,

(2.b): $(\alpha / \beta)_{S} /(\alpha / \beta)_{T}=1$,

$(2 c):(\alpha / \beta)_{S} /(\alpha / \beta)_{T}=0.05$.

With reference to Figs. 2., typical $(\alpha / \beta)_{S}$ values for survival of human cells exposed to low-LET $\gamma$ rays are around $10 \mathrm{~Gy}$, whereas the approximation of Eq. (7) is reasonable for values of $(\alpha / \beta)_{S}$ greater than 20-25 Gy. Thus the approximation of Eq. (7) does not in general hold well at low LET.

Because we have assumed that $\beta$ is constant, increasing values of $(\alpha / \beta)_{S}$ in Figs. 2 correspond to increasing values of $\alpha_{S}$, i.e. to increasing $\mathrm{RBE}$, and therefore to increasing LET. For low-LET $\gamma$ rays, $(\alpha / \beta)_{S}$ is around 10 Gy. As LET increases, $(\alpha / \beta)_{S}$ increases, until at values of $(\alpha / \beta)_{S}$ greater than 20-25 Gy, corresponding to RBEs greater than about 2-2.5, the approximation of Eq. (7) does hold, i.e. the maximum oncogenic potential is virtually constant. Depending on the cell line, radiations that produce RBEs for survival greater than 2-2.5 have LETs which are greater than around $20-40 \mathrm{keV} / \mu \mathrm{n}$. From this we conclude that the maximum possible rate of 
oncogenesis is, in general, independent of LET for all but sparsely-ionizing radiations.

In perhaps the most extensive study of carcinogenesis in vivo for different high-LET radiations, Fry et al (1985) concluded in relation to induction of mouse Harderian-gland tumors that "...the curves for all the radiation qualities appear to plateau at a similar prevalence level...", which is in agreement with the predictions of Eq. (7).

From Fig. 2, it can be seen that the maximum oncogenic potential of high-LET radiations can be greater than, equal to, or smaller than that for low-LET radiation depending on the relative values of $(\alpha / \beta)_{S}$ and $(\alpha / \beta)_{T}$. If $(\alpha / \beta)_{T}$ is larger, then high-LET radiation will have a greater maximum oncogenic potential than low LET; if $(\alpha / \beta)_{S}$ is larger, then low-LET radiation will have a greater maximum oncogenic potential (MOP); if the ratios are the same, high-LET and low-LET radiation would have the same MOP.

For densely-ionizing radiations, oncogenic induction generally reaches a maximum at doses in the region of $0.5 \mathrm{~Gy}$ - and then either remains constant or decreases slowly with increasing dose. Thus the conventional system of risk estimation, in which risk is assumed to be proportional to dose, is inappropriate at such doses (or higher) of densely-ionizing radiation. In a situation where at least a sufficient dose has been delivered to attain the maximum possible rate of oncogenesis, this maximum oncogenic potential (MOP) appears to be a natural quantity on which to base an assessment of risk. It is a conservative quantity in that no presumption is made concerning decreasing risk as the dose is increased beyond that producing the maximum oncogenic potential.

Both experimental and theoretical considerations imply that, for densely ionizing radiations, this maximum oncogenic potential (MOP) is independent of LET. These observations lead to a rather simple risk-assessment system for doses above about $0.5 \mathrm{~Gy}$ of densely-ionizing radiation $(220-40 \mathrm{keV} / \mu \mathrm{m})$. Again, it is stressed that it is not necessarily the risk per se, which is independent of LET and, of course, dose, but rather the maximum-possible risk, the MOP.

We estimate that the MOP should approach a constant value when the low-dose RBE for survival, relative to $\gamma$ rays, reaches a value of 2-2.5, corresponding to LETs of $\geq 20-40$. This LET estimate is quite conservative in that it has often been suggested that the low-dose RBE for $250-\mathrm{kVp} \times$ rays (track-averaged LET $\sim 1.5 \mathrm{keV} / \mu \mathrm{m}$ ) relative to megavoltage $\gamma$ rays (track-averaged LET $\sim 0.25 \mathrm{keV} / \mu \mathrm{m}$ ) is $1.5-2$. If this is the case, then the LET at which the maximum oncogenic potential reaches a constant value may be as low as $3-10 \mathrm{keV} / \mu \mathrm{m}$.

It is an open question as to how an estimate of this "single" high-r. ET MOP can be made which is applicable to man. (Of course the MOP is only unique for a given oncogenic endpoint). In the light of the reassessment of the Japanese A-bomb dosimetry, there is little whole-body human exposure at high LET on which to 
draw. The high-exposure cohorts of the Colorado uranium miners exposed to radon-daughter alpha particles may be the most appropriate data source.

The implications of this risk assessment system would primarily be for the radon problem, which is the dominant source of collective high-LET dose. Epidemiological and in-vivo data on lung-tumor induction by prolonged exposure to radon daughters suggest that the lung-cancer risk starts to level off at a cumulative exposure of around 1000 WLM (Working Level Months, corresponding to an effective dose of $\sim 0.6 \mathrm{~Gy}$ ). It would follow that low-exposure environmental risk estimation should be based on data at considerably lower exposures than 1000 WLM. For comparison, the average cumulative exposure of the Colorado uranium miners analyzed in the BEIR-IV report was around 500 WLM and, in that report, radon risk estimation was based on the cohort with cumulative exposures of 2000 WLM or less.

Based on extrapolated survey data, an estimated 2000 homes in the U.S.A. may have radon levels above $65 \mathrm{pCi} / \mathrm{l}$, which could yield lifetime exposure levels of more than 1000 WLM. The risk-estimation system under consideration here would be directly applicable to this population.

Another area of application would be in cancer risk assessment related to an manned interplanetary space mission. In such a scenario, where a dose of at least 0.5 Gy of mixed high-LET radiations would be delivered, concerns about the precise dose and energy spectrum of cosmic radiation to which the astronauts would be exposed may not be necessary.

For sparsely-ionizing radiation, the maximum oncogenic potential will not, in general, be attained until a dose of several Gray is delivered; at such doses, acute effects would be more important than the carcinogenesis risks discussed here. At doses below several Gray, the conventional system of linear risk estimation would still be the most appropriate. We consider, therefore, that the MOP concept would have little application to low-LET risk assessment.

In conclusion, we suggest that at doses greater than about 0.5 Gy of densely-ionizing $(\geq 20-40 \mathrm{keV} / \mu \mathrm{m})$ radiation, it is unreasonable to assume that the risk increases linearly with increasing dose. For densely-ionizing radiations above $\sim 0.5 \mathrm{~Gy}$, a risk estimation system based on the maximum possible oncogenic transformation rate (MOP) would a) be more credible than the assumption of a risk that increases linearly with dose, b) be appropriately conservative and c) be simple and practical to use. 


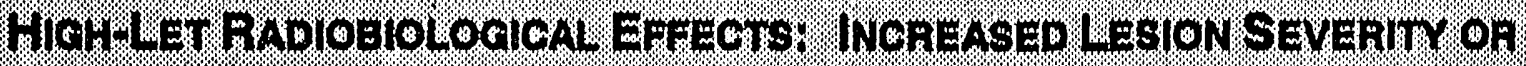 TMCREASED L ESTON PROXIMIV?}

\section{J. Brenner (In Collaboration with R. K. Sachs, Physics Department, Unlversity of Callfornla, Berkeley).}

Exchange-type chromosomal aberrations produced by ionizing radiation are probably caused by the pairwise interaction of DNA double-strand breaks (DSB). It is not, however, known whether all DSB (e.g. as measured by neutral elution) are likely candidates for producing exchanges, or only a specific subset (e.g. severely-damaged long-lived DSB, or those measured as PCC breaks). We suggest that not only the yield, but also the variance, of exchange-type aberrations can provide significant constraints on models describing their formation.

A useful "test bed" for such models is the effect of high-LET radiation. As an example, the increased biological effectiveness of high-LET radiation can be ascribed either to a) the increased proximity of DSB with increasing LET or b) an increased frequency of severely-damaged (complex) DSB. In the latter case, proximity considerations would be of lesser importance because DSB restitution would be unusually slow, implying that the initial spatial distribution could be lost before exchange-type interactions take place.

Modeling long-lived DSB, in which exchange dominates restitution, can give quite different results than are obtained by making the usual assumptions that only a small minority of DSB participate in exchanges. For example, in the limiting case of no restitution at all, if the induction of the long-lived DSB is linear with dose the induction of complete exchanges will also be approximately linear. This contrasts with the quadratic dose dependence for inter-track interactions inferred from the usual assumption that restitution dominates exchange. Assuming exchange dominates restitution also typically leads to smaller theoretical estimates of the variance.

For the case of slowly-restituting DSB, the implied spatial homogeneity allows the use of Markov models which, together with microdosimetric information, have been used to model the yield and variance of dicentrics produced by irradiation of $\mathrm{G}_{0}$ human lymphocytes with various radiations. The effects of interphase death were also modeled.

From comparisons with the data, we conclude that long-lived DSB could make a significant contribution to the observed high-LET dicentric yield (at all doses), as well as the observed low-LET dicentric yield (at low doses). High-dose low-LET yields, however, are clearly not a result of this mechanism. On the other hand, Monte-Carlo based simulations, based on proximity explanations of high-LET effects, result in some uniformity of the key parameters amongst different kinds of radiations, consistent with evidence from survival studies that there are common mechanisms for low, medium, and high-LET radiation damage. 
Overall, it appears that proximity effects and the effects of severely-damaged DSB could produce similar results at high LET. Variance measurements hold out some possibility of distinguishing between these mechanisms.

Experimentally, no direct detection of "severely damaged" DSB has been reported. Relevant evidence comes from experiments by Cornforth, who measured the yield of chromosome terminal deletions in human skin fibroblasts in various stages of the cell cycle, at low and high LET. If a terminal deletion is interpreted as resulting from a DSB so severely damaged that it never rejoins, then models predicting that severely-damaged DSB also dominate exchange formation at high LET would predict more terminal deletions for alpha particles than for $x$ or gamma rays. In fact the opposite is observed, which might be taken as evidence supporting the "proximity" theory of DSB interaction. 
CELLULAR AND MOLECULAR BIOLOGY 


\section{Photoneutrons generated by High Energy Medical Linacs}

\section{Erlc J. Hall}

The production of photoneutrons in high-energy medical Linacs is a subject of considerable topical interest, and a real challenge to the neutron dosimetrist. More and more high-energy machines of around 18 to $20 \mathrm{MeV}$ or higher are in routine clinical use for radiation therapy, particularly in treatment plans designed with curative intent; this is because of the superior dose distributions that can be obtained for deep-seated tumors. Photoneutrons are generated, primarily, in the high-Z material of the collimators, with a threshold of about 6 to $8 \mathrm{MeV}$ for lead, tungsten or uranium. There is a giant resonance (i.e. a peak) in the photoneutron production cross section in these heavy materials at a photon energy around 14 $\mathrm{MeV}$.

Neutrons with a broad energy range are generated, the mean being around 1-3 $\mathrm{MeV}$. Various attempts have been made to measure the quantity and quality of these neutrons, against the background of high-energy $x$-rays. The most elegant attempt to date utilized an ultra-miniature tissue-equivalent proportional counter (Kliauga, personal communication, 1991). Neutrons are emitted isotropically and contribute about 0.1 to $0.3 \%$ by dose to the primary $x$-ray field; because of their range, this represents a whole-body neutron dose to the patient.

Medium-energy neutrons are highly effective biologically, and so it is of interest to make an assessment of the impact of photoneutrons on radiotherapy in terms of the possible induction of second malignancies:

Assuming a treatment dose of $6000 \mathrm{cGy}$ over 6 weeks, a neutron dose of 0.1 to $0.3 \%$, with a quality factor 10 to 20 for the mixture of neutron energies (some of which would qualify for 20 and some for 10 according to ICRP), then the whole body dose equivalent to the patient would amount to:-

$$
6000 \times \frac{(0.1 \text { to } 0.3)}{100} \times(10 \text { to } 20) \mathrm{cSv}=60 \text { to } 360 \mathrm{cSv}
$$

Using a risk estimate of about $3 \%$ per Sv for protracted whole-body irradiation (based on BEIR V and UNSCEAR), this whole-body dose would produce an incidence of second malignancies of about 2 to $10 \%$. This is, of course, in addition to the risk from the photon dose, which would not be significantly different from that characteristic of lower-energy Linacs or cobalt teletherapy units.

In the United States, about one million new cases of cancer are reported annually, about half of which are treated with radiotherapy. Half of these are treated with intent to cure, and about half of these are cured. This amounts to about $1 / 8 \times 10^{6}$ long-term survivors per year. If about $1 / 3$ of these patients were treated with high energy linacs, the number of second malignancies that might be induced by photoneutrons would be:- 
( 2 to $10 \%) \times 1 / 3 \times\left(1 / 8 \times 10^{6}\right)=800$ to 4000

This estimate is for the United States, and there would be a comparable number in Europe, as more high energy Linacs come into routine clinical use. These secondary malignancies are the price for an improved dose distribution which, presumably, leads to better tumor control and/or reduced normal tissue morbidity. It might seem to be a high price that needs to be considered further. Indeed these calculations have spawned a project to measure the biological effectiveness of photoneutrons using in vitro assays for oncogenic transformation. 


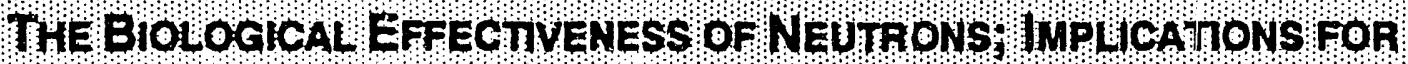 \\ RADLATON RHOTECTION}

\section{Eric J. Hall and David J. Brenner}

The radiobiology of neutrons has important societal implications. In nuclear facilities world-wide, several hundred thousand individuals are monitored as potentially receiving doses of neutrons, while there is increasing concern about the dose to which airline crew members are exposed, which includes a substantial neutron component. This represents an even larger number of individuals.

It is this of considerable interest to understand the biological hazards posed by these radiations, and to be able to relate them to the better-understood hazards of $x$ rays.

A major problem in understanding such hazards is the lack of human data. The recent reassessment of radiation doses delivered during the A-bomb attacks on Hiroshima and Nagasaki have resulted in a major reduction in the estimated neutron doses at Hiroshima compared with that suggested by the old "T65D" dosimetry.

Thus, any estimates of the biological hazards posed by neutron exposures must be based, to a significant degree, on in-vitro radiobiological experiments, and on experiments with animals.

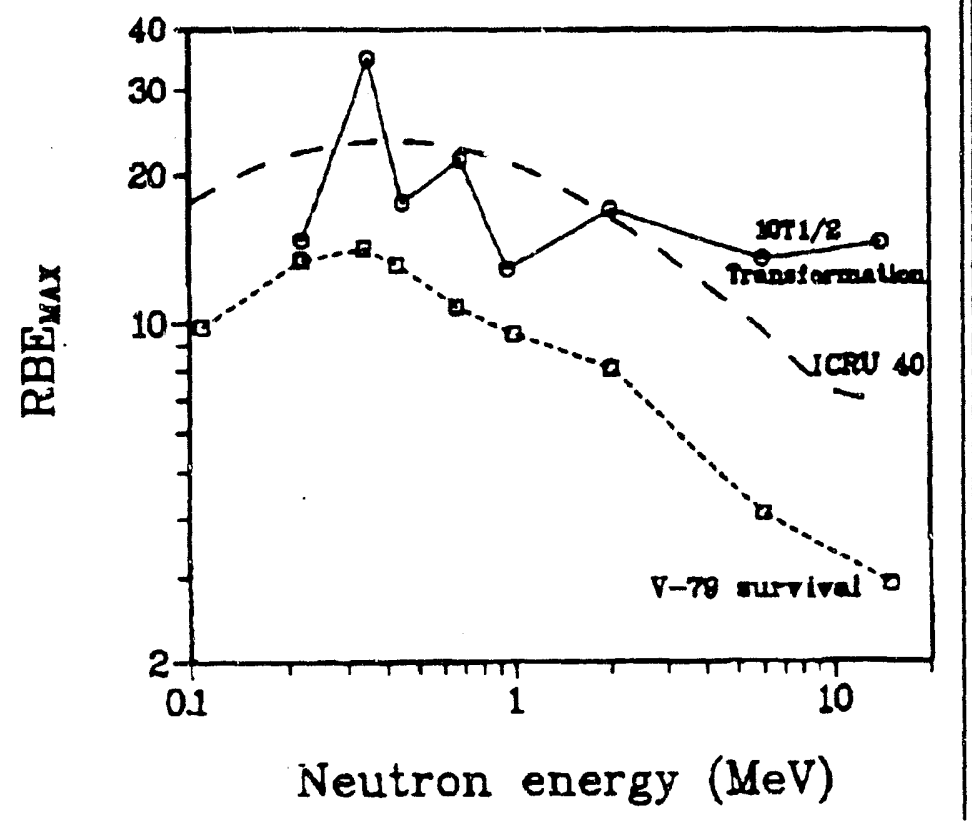

Fig. 1: Relative Biological Effectiveness (RBE) as a function of neutron energy as measured by different biological endpoints. The transformation data, obtained with $\mathrm{C} 3 \mathrm{H}$ 10T1/2 cells, are from Miller et al 1989. The cell lethality data obtained with V79 Chinese hamster cells are from Hall et al 1975. The dotted line represents values of $\bar{Q}$ suggesied in iChu report 40(1986).
The problem, then, is tc generate appropriate values of $W_{R}$ for the various neutron fields (at appropriate doses and dose rates) to which workers are exposed.

Figure 1 shows $\mathrm{RBE}$ as a function of neutron energy from 0.1 to $15 \mathrm{MeV}$, based on survival of V79 Chinese hamster cells and oncogenic transformation using $\mathrm{C} 3 \mathrm{H}$ $10 \mathrm{~T} 1 / 2$ cells as an in vitro assay.

There is broad agreement, particularly in shape, between the various data sets, within the limits of experimental error which is considerable, especially in the case of the transformation data. It is of interest to note that $\mathrm{RBE}_{\mathrm{M}}$ values for 
transformation are in close accord with the $Q$ values suggested by ICRU 40 report.

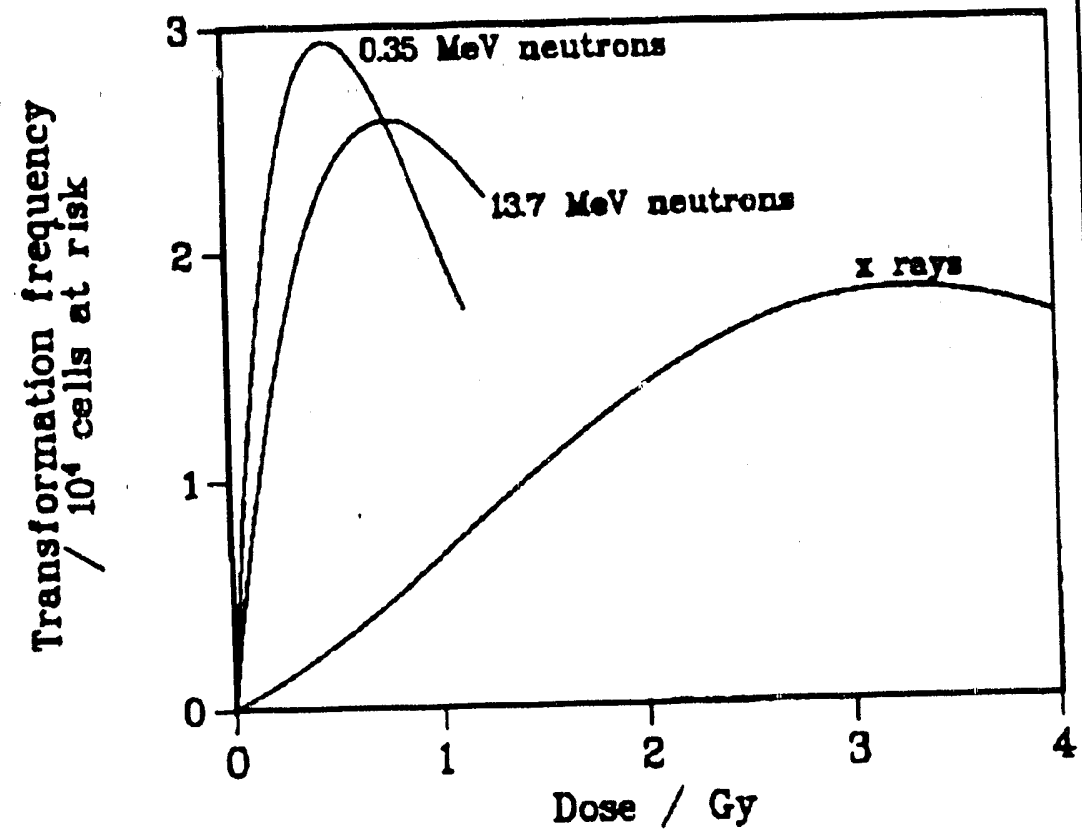

Transformation data are usually plotted in terms of transformation incidence per surviving cell. However, for extrapolating in vitro data to an in vivo situation where a whole organ or organism is exposed, a more relevant quantity may be transformation incidence per initial cell at risk. This quantity is shown as a function of dose for $x$-rays and for two neutron energies in Figure $2 . \quad$ The transformiation incidence per initial cell

Fig. 2: Transformation frequency per initial cell at risk as a function of dose for $\mathrm{C} 3 \mathrm{H} 10 \mathrm{~T} 1 / 2$ cells exposed to x-rays and to neutrons of 0.35 and 13.7 MeV. Transformation frequency per initial cell at risk rises rapidly with dose at low doses, reaches a peak, and then falls as cell killing takes over. Note that the peak rises to a higher value, and occurs at lower doses as the neutron energy decreases. Calculations based on data in Miller et al 1989.

killing takes over. There are three points of interest:at risk rises rapidly with dose at low doses, reaches a peak and then falls off rapidly with dose at higher doses, as transformation saturates and cell

- The transformation incidence per initial cell at risk reaches a somewhat higher value for a neutron dose of 30 to $50 \mathrm{cGy}$ than for any dose of $\mathrm{x}$-rays, however large.

- The peak incidence gets progressively larger for lower energy neutrons.

- The peak incidence occurs at a progressively lower dose as the neutron energy falls and the effectiveness per cGy of the neutrons increases.

\section{References}

Hall, E.J., Novak, J.K., Kellerer, A.M., Rossi, H.H., Marino, S. and Goodman, L.J. $\mathrm{RBE}$ as a Function of Neutron Energy 1. Experimental Observations Radiation Research 64 245-255 (1975)

International Commission on Radiation Units and Measurements. The Quality Factors in Radiation Protection. Bethesda, MD: ICRU Report 40, (1986) 
Miller, R.D., Geard. C.R., Brenner, D.J., Komatsu, K., Marino, S.A. and Hall, E.J. Neutron-Energy-Dependent Oncogenic Transformation of $\mathrm{C} 3 \mathrm{H}$ 10T1/2 mouse cells. Radiation Research, 117 114-127 (1989). 


\section{Greg A. Freyer, Yong Yu and Julle Folnsteln}

Having developed a general scheme for the isolation and identification of oncogenes based on a gamma-ray transformed $\mathrm{C} 3 \mathrm{H} 1011 / 2$ cell line, we have now focused on the isolation and identification of a neutron induced oncogene. To this end we are studying a cell line derived from a C3H 10T1/2 cell transformed by 60 cGy of $6 \mathrm{MeV}$ neutrons. This cell line is characterized by anchorage independent growth and the formation of tumors in mice. High molecular weight DNA was isolated from this cell line. The DNA was partially digested with Mbo I and size selected. Two of the four base 5 ' overhang of each end was filled in with DNA Polymerase I (large fragment) using dGTP and dATP. The cosmid, pWE15XhoI, was digested with XhoI and two of its four base 5 ' overhang filled in with dCTP and dTTP. The DNAs were mixed in a molar ratio of 1:1 and ligated. The ligation mixture was packaged into lambda phage particles and used to infect A490 cells. Transformants were selected by growing in the presence of ampicillin. The library contains approximately $10^{6}$ individual clones. The library has been tested for background and average insert size.

Total library DNA was then transfected into normal C3H $10 \mathrm{~T} 1 / 2$ cells with transfectants selected using G418. In the past cells were plated at a density such that approximately 200-500 colonies would grow on each plate. This dilution is necessary in order to be able to detect foci in this system. However, this has meant screening as many as 1500 plates in order to identify one or trvo foci. With this approach there is a good chance that transformed colonies are missed. For this reason, we are currently testing an alternative strategy for identifying and isolating a transformed clone. Following transfection of total library DNA into normal $\mathrm{C} 3 \mathrm{H}$ $10 \mathrm{~T} 1 / 2$ cells, the cells are plated at a density such that approximately $2000-5000$ G418 resistant colonies grow on each plate (approximately 10 fold higher density then previously used). Once the cells reach confluence they remain on the plates for an additional seven days. At this point foci are not obvious. The cells from all of the plates are trypsinized, washed and made into a single pool. Several aliquots of the cells are frozen for future use while the remainder are used to identify transfected cells which have picked up an activated oncogene. To do this, approximately $10^{6}$ cells are injected at each of two sites into mice which were previously exposed to $4 \mathrm{~Gy}$ of whole body gamma-rays. The mice are checked periodically for the occurrence of tumors.

Once tumors are detected they will be isolated and expanded. High molecular weight DNA will be isolated from the tumor cell lines. The DNAs will be digested with Hind III and electrophoresed on agarose gels. The gel will be blotted onto a nylon membrane. The membrane will be probed with a restriction fragment from the vector pWE15. This will allow us to determine the number of cosmids present in each clone. Typically this number is around 15-30. To obtain a transformed cell line with a single oncogene, secondary transformants need to be obtained. This is 
typically done isolating total genomic DNA from the transformed cell line and transfecting it into normal cells. This is a very inefficient process with C3H 10T1/2 cells. Recently we have employed a different strategy to obtain secondary transformants. Transformed cells are irradiated on ice with $120 \mathrm{~Gy}$ of gamma rays. This kills the cells and fragments its DNA. These cells are then fused with normal $\mathrm{C} 3 \mathrm{H} 10 \mathrm{~T} 1 / 2$ cells. To identify clones which have picked up DNA fragments containing cosmid sequences, the fused cells are selected in G418. These cells are then tested for type III focus formation. DNA isolated from these cells are again probed with a fragment of vector DNA to determine the cosmid copy number. Cell lines containing a single cosmid sequence are then used to isolate the oncogene by cosmid rescue. The isolated sequence will be characterized, first by its ability to hybridize with known oncogene sequences. If this does not identify the oncogene its exons will be identified (by isolating a cDNA or by exon trapping) and sequenced.

Preliminary studies suggest that this is a sound strategy and experiments with our neutron transformed cell line are currently under way. In addition we have isolated several other neutron transformed cell lines. These have been transformed with neutrons with a range of energies from $230 \mathrm{keV}$ to $14 \mathrm{Mev}$. 


\title{
THE INVERSE DOSE-RATE EFFECT FOR ONCOGENIC
}

\section{TRANSFORMATION BY CHARGED PARTICLES IS LET DEPENDENT}

\author{
Richard C. MIller, Gerhard Randers-Pohrson, \\ Ludwig Hleber, Stephen A. Marino, Marcla Rlchards, and
}

Eric J. Hall

Radiation workers in certain areas of the nuclear power industry, shuttle astronauts in low earth orbit, as well as the crews of commercial jet airliners are exposed over an extended period of time to low doses of ionizing radiation that includes a substantial proportion of intermediate to high LET (Linear Energy Transfer).

For most cellular endpoints, lowering the dose-rate, or protracting the exposure over a longer time period by fractionation, leads to a reduction or sparing in effect, at least for $X$ or gamma rays.

An exception to this generalization is the anomalous "inverse dose-rate effect" observed for oncogenic transformation induced by neutrons. Hill et al. (1982) first reported this effect for $\mathrm{C}_{3} \mathrm{H} \quad 10 \mathrm{~T}_{1 / 2}$ cells exposed to modified fission-spectrum neutrons at the Janus reactor. They showed that the incidence of transformation induced by a given dose of neutrons was enhanced if the dose was given continuously at low dose rate, or delivered in a series of dose fractions over 5 days compared with a single acute exposure. In recent years, several dose-rate studies using $\mathrm{C}_{3} \mathrm{H}$ 10T $_{1 / 2}$ cells have appeared in the literature with some supporting the existence of an inverse dose-rate effect, and others failing to observe it. Miller et al. (1988) suggested that the enhancement of transformation by fractionation was neutron-energy dependent. In summarizing and analyzing all of the data available for this cell line, Brenner and Hall (1991) came to the conclusion that the inverse dose-rate effect required a subtle combination of dose, dose-rate and the LET of the radiation.

The present report describes experiments designed to investigate the LET dependence of the inverse dose-rate effect using charged particles of defined LET in the track segment mode.

Exponentially growing $\mathrm{C}_{3} \mathrm{H} 10 \mathrm{~T}^{1 / 2}$ clone 8 cells were plated onto thin-mylar bottomed dishes $(6 \mu \mathrm{m})$ to allow penetration of the charged particles. Immediately after the final irradiation, cells were trypsinzed and replated into $100-\mathrm{mm}$-diameter tissue culture petri dishes. Cells were grown in Eagle's basal medium with $10 \%$ heat-inactivated calf serum supplemented with iron and $25 \mu \mathrm{g} / \mathrm{ml}$ gentamycin. The batch of serum selected for use resulted in growth and transformation characteristics similar to previous batches used. At the end of the incubation period, cells were fixed in formalin and stained with Giemsa. Cell survival was determined by the colony assay method while transformed foci types II and III were identified 
according to criteria described by Reznikoff et al. (1976). Irradiations with charged particles were performed using the track segment facility at the Radiological Research Accelerator Facility (RARAF) of Columbia University.

Cells were exposed to 25-, 40-, 75-, 90-, 120-, 150-- and 200-keV/ $\mu \mathrm{m}$ charged - particles. Total doses were $0.6 \mathrm{~Gy}$ for $25 \mathrm{keV} / \mu \mathrm{m}$ exposures, $0.3 \mathrm{~Gy}$ for $40 \mathrm{keV} / \mu \mathrm{m}$ and $0.2 \mathrm{~Gy}$ for the remainder of the high LET particles and were selected to elicit similar levels of cell killing (71-82\% survival). For each radiation quality, the doses were delivered in three equal fractions at intervals of $0.3,1.5,15,45$, and $150 \mathrm{~min}$. Three dishes were used for each treatment.

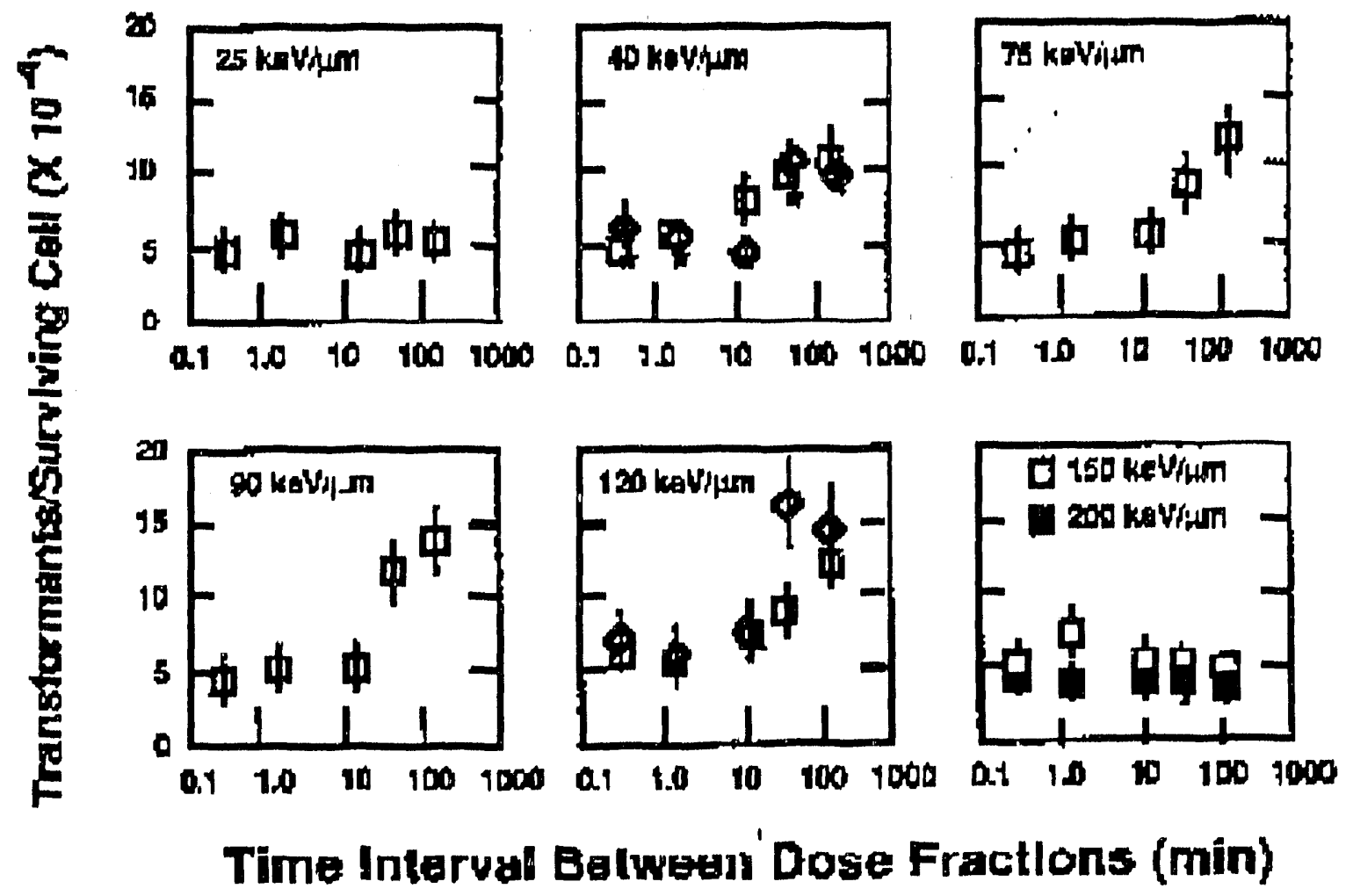

Fig. 1 Induced oncogenic transformation of $\mathrm{C}_{3} \mathrm{H} 10 \mathrm{~T} 1 / 2$ cells by 25-, 40-, 75-, 90-, 120-, 150- and 200- $\mathrm{keV} / \mathrm{um}$ charged particles as a function of time between dose fractions. Total doses were selected at each radiation quality to obtain similar levels of cell killing. Error bars represent $\pm 1 S D$.

Figure 1 shows the accumulated data for cells irradiated with seven different LETs, each delivered in 3 dose fractions, with various time intervals between the fractions. The precise doses used are shown in the protocol. Enhancement of transformation incidence is seen only for LET values between 40 and $120 \mathrm{keV} / \mu \mathrm{m}$, and the maximum enhancement of transformation incidence is evident when the interfraction interval is about 150 minutes.

The data of Figure 1 are summarized in Figure 2 in which the maximum change in transformation incidence caused by fractionation is plotted as a function of LET. Plotted in this way, the data clearly demonstrate that the inverse dose-rate effect i.e., 
the enhancement of transformation by fractionation, occurs only over a limited range of LET values. No enhancement is seen for radiations of low or very high LET'.

The experiments reported in this paper, using charged particles of defined LET, demonstrate convincingly that the "inverse dose-rate effect," i.e., the enhancement of transformation incidence when a dose of radiation is protracted either by fractionation or by low dose-rate, is LET dependent. Only LETs in the range between 40 and $120 \mathrm{keV} / \mu \mathrm{m}$ produce an enhanced effect due to fractionation; radiation of either lower or higher LET does not. It is further supported by the results of Bettega et al. (1992) who showed an enhanced effect of fractionation by 4.3 $\mathrm{MeV}$ alpha particles (LET $101=\mathrm{keV} / \mu \mathrm{m}$ ), and also by the results of Hieber et al. (1991) who demonstrated enhancement by fractionation with alpha particles having an LET of $100 \mathrm{keV} / \mu \mathrm{m}$ but not with alpha particles having an LET of $147 \mathrm{keV} / \mu \mathrm{m}$ (1987).

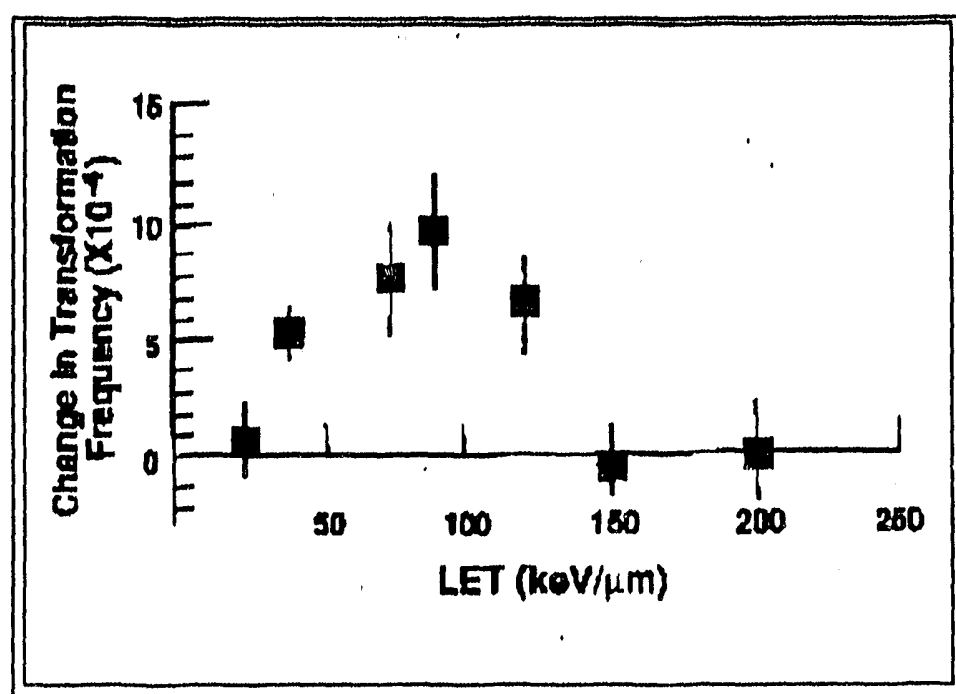

Fig. 2 Change in transformation frequency for $\mathrm{C}_{3} \mathrm{H} 10 \mathrm{~T} 1 / 2$ cells irradiated for the minimum (0.3 $\mathrm{min}$.) and maximum $(150 \mathrm{~min}$.) fractionation intervals as a function of LET. Error bars represent \pm 1 SD.
Rossi and Kellerer (1986) sought to explain the inverse dose-rate effect in terms of a biophysical model. They assumed a "window of sensitivity" to radiationinduced transformation at some point in the cell cycle. If this is the case, an acute exposure of cycling cells to high-LET iadiation will result in some fraction of these sensitive ceils receiving (on average) very large depositions of energy - much greater than required to produce the changes that may lead to oncogenic transformation. On the other hand, if the exposure is protracted or
fractionated, a larger proportion of sensitive cells will be exposed, though to smaller average numbers of energy depositions. However, because exposures to mediumor high-LET radiations are involved, it was postulated that the total specific energy deposited in the sensitive cells by the smaller number of energy depositions will still be sufficient to produce a potentially oncogenic change in the cell. To the extent that this latter postulate may not hold at low LET, the inverse dose-rate effect would not be expected to apply to such radiations, which agrees with experimental observations. 


\section{PUBLLCations}

Brennex, D.J., Significance of neutrens from the atomic bombs at Hiroshima for revised radiation risk estimates. Health Physics, $\underline{60}$ 439-442, 1991

Brenner, D.J., Medvedovsky, C., Huang, Y., Merrium, G.R., and Worgul, B.V., Accelerated heavy particles and the ions VI. RBE studies at low doses. Radiation Research, 128, 73-81, 1991

Hall, E.Y., Miller, R.C., Brenner D.J., Neoplastic transformation and the inverse doserate effect for neutrons. Radiat. Res., 128, 575-580, 1991

Hall, E.J., Fuilla Memorial Lecture, From beans to genes - back to the future. Radiation Res., 129, 235-249, 1992

Kliauga, P., Techniques for measurement of energy deposition in nanometer volumes. Radiation Research: A Twentieth Century Perspective, 409-414, Ed. by W.C. Dewey et al., Academy Press, New York, 1992

Miller, R.C., Geard, C.R., Marino, S.A., Richard, M. and Randers-Pehrson, G., Oncogenic transformation following sequential irradiations with monoenergetic neutrons and x-rays. Radiation Research, $125,338-342,1991$

Musolino, S.V., McGinley, P.H., Greenwood, R.C., Kliauga, P. and Fairchild, R.G., Evolution of an iron-filtered epithermal neutron beam for neutron-capture therapy. Med. Phys., 18, 806, 1991

Rossi, H.H. and Zaider, M., Neutron RBE for A-Bomb survivors. RERF Update 3 , 34, 1991

Rossi, H.H. and Zaider, M., Elements of microdosimetry. Med. Phys., 18, 1085, 1991

Rossi, H.H., Alsmiller, R.G., Berger, M.J., Kellerer, A.M., Roesch, W.C., Spencer, L.A. and Zaider, M., Conceptual basis for calculations of absorbed-dose distributions. NCRP Report, 108, 1991

Rossi, H.H. and Zaider, M., Compound Dual Radiation Action. Radiat. Res. (in press)

Vracko, M.G. and Zaider, M., A calculation of the exciton energies in periodic systems with helical symmetry: Application to a hydrogen fluoride chain. Int. J. Quantum chem., (in press)

Zaider, M., Charged-particle transport in the condensed phase. In: Physical and Chemical Mechanisms in Molecular Radiation Biology. (In press, 1991)

Zaider, M., The interaction of two agents revisited: response to M.C. Berenbaum. Radiat. Res., 126, 266-268, 1991

Zaider, M. and Rossi, H.H., On the question of reverse RBE. Radiat Res. (In press) 
Zaider, M., Evidence of a neutron RBE of 70 for solid-tumor induction at Hiroshima and Nagasaki and its implications for assessing the effective neutron quality factor.

Health Phys., 61, 631-636, 1991 

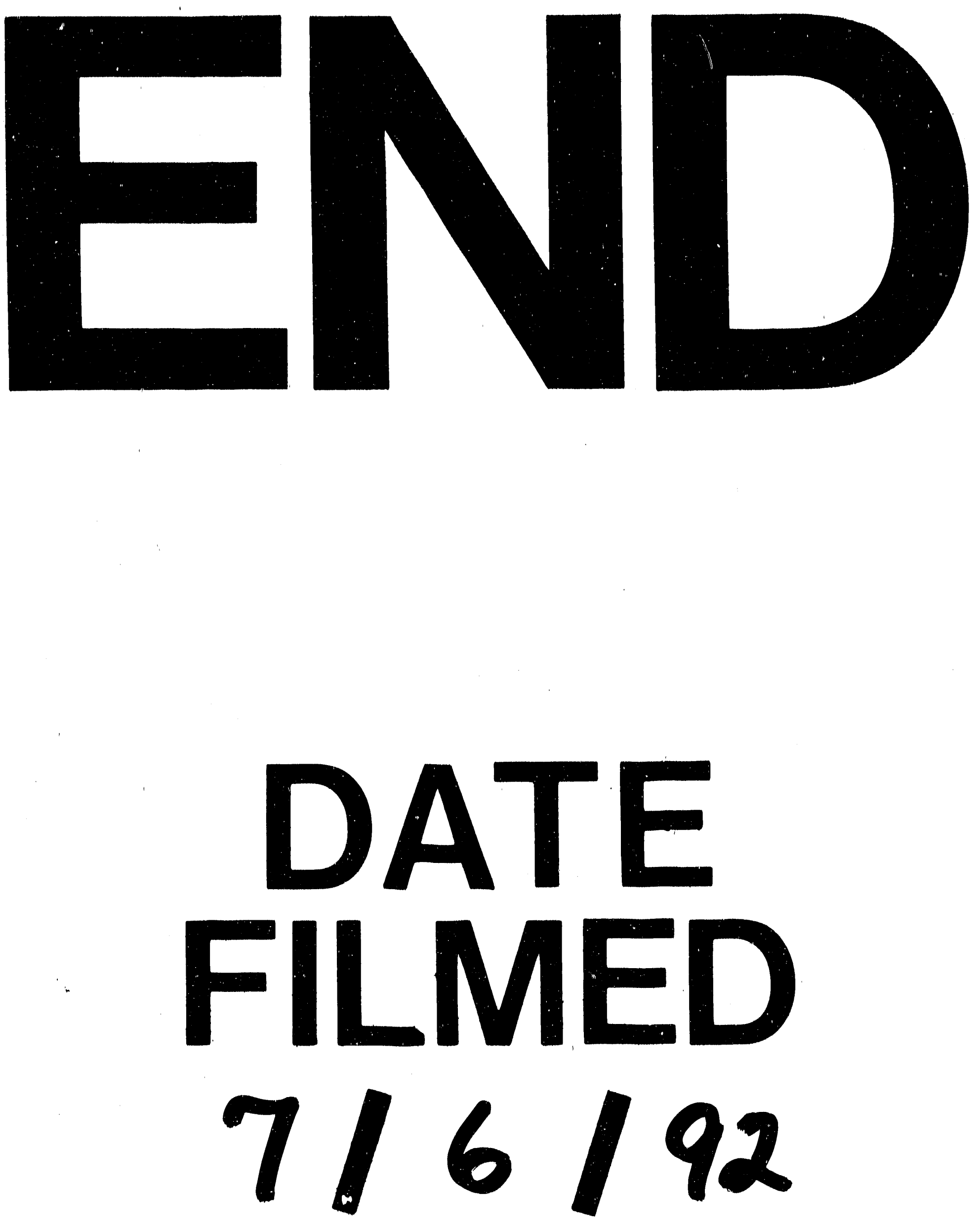
